\title{
Fisheries Management Under Cyclical Population Dynamics
}

\author{
Richard T. Carson - Clive W. J. Granger • \\ Jeremy B. C. Jackson • Wolfram Schlenker
}

Received: 6 August 2008 / Accepted: 2 December 2008 / Published online: 24 December 2008

(C) The Author(s) 2008. This article is published with open access at Springerlink.com

\begin{abstract}
Cycles in environmental conditions (e.g., sea-surface temperature) directly impact fish growth. This paper extends the classical Gordon-Schaefer fishery model by replacing the constant growth rate with a cyclical growth rate. The optimal harvest rate is shown to fluctuate, but the cycle of the harvest rate lags the cycle of the biological growth function with the highest harvest rate occurring after biological conditions start to decline. Simulations contrast various fishing policies and illustrate the proclivity to crash a fishery if it is wrongfully managed as if there is a constant growth rate with i.i.d. environmental shocks. Finally, we show that small cyclical fluctuations in one species can result in large fluctuations in the optimal harvest rate of another species if the fish species are interlinked through predator-prey relationships.
\end{abstract}

Keywords Cyclical growth parameters · Environmental fluctuations · Gordon-Schaefer model

JEL Classification Q22 - D78

R. T. Carson - C. W. J. Granger - J. B. C. Jackson

University of California, San Diego, USA

e-mail: rcarson@econ.ucsd.edu

C. W. J. Granger

e-mail: cgranger@ucsd.edu

J. B. C. Jackson

e-mail: jbjackson@ucsd.edu

W. Schlenker $(\varangle)$

Columbia University and NBER, New York, USA

e-mail: wolfram.schlenker@columbia.edu 


\section{Introduction}

Reports of two recent high level commissions, one sponsored by a major foundation (Pew Oceans Commission 2003) and one by the US government (U.S. Oceans Commission 2004), suggest that serious problems exist with respect to the management of fisheries. Many fisheries are clearly overfished and in serious decline. Conventional wisdom is that political considerations have prevented rational management of fisheries by allowing overfishing, failing to curtail increases in fishing capacity, and not taking steps to prevent ecosystem damage by fishing selective species or size classes. While international fisheries are a classical example of an open-access resource, the extension of exclusive fishing zones out to 200 miles around countries in the mid-1970s appropriated large parts of fisheries to individual nations. It is estimated that more than $90 \%$ of all fish reside within this 200 -mile zone as coastal regions offer a more ample food supply for fish (Bjorndal and Munro 1998). With such a large fraction of the fish stock within the jurisdiction of individual countries, it becomes an interesting question why national fisheries regulation have not been more successful at managing costal zone fisheries.

Standard economic fishery models with autonomous growth parameters predict that the optimal policy should be a constant target stock. Under such a policy the regulator increases/ decreses the allowable harvest quantity to offset any deviations from the target stock. While political rent seeking considerations without doubt are an important factor why fisheries regulation have failed to prevent significant over-fishing in the past, we show that a traditional constant target stock can be suboptimal when the growth function is in fact cyclical.

Cyclical growth parameters have been observed in various species, e.g., the cod stock. Hannesson and Steinshamn (1991) argue that "the Arcto-Norwegian cod is not unique in this respect; many and probably most fish stocks exhibit such fluctuations, but possibly with a lesser amplitude and regularity."1 A classical book on fish stock assessment by Hilborn and Walters (1992, p. 52) shows that the recruitment of Pacific halibut between 1945 and 1985 appear to follow predictable synodal cycles. Such fluctuations in recruitment will result in varying fish densities, and hence, fluctuating values of fishing licenses along the lines of Stefanou and Wilen (1992). One of the biological reasons behind fluctuating growth parameters are cyclical ocean temperatures and salinity levels that influence fish growth: for example, sea surface temperatures in the Pacific Ocean exhibit strong cyclical components. The El Nino Southern Oscillation (ENSO) is of moderate duration, while the Pacific Decadal Oscillation is on the order of 50 years. Chavez et al. (2003) link the fluctuations in sardine and anchovies cycles to fluctuations in the Pacific Decadal Oscillation. Arnason (2006) emphasizes that climate change is predicted to change ocean temperatures, and hence, biological growth parameters with unknown effects on species interactions. Of course, overfishing and adverse environmental conditions may occur together. For example, though still controversial, there is some indication that the Peruvian anchoveta collapsed because high fishing efforts overlapped with environmental events that resulted in prolonged low growth rates (Ludwig et al. 1993).

The contribution of this paper is to relax the classical modeling assumption of a timeinvariant biological growth function in a Gordon-Schaefer fishery model and replace it with a periodically oscillating growth function. This is different from most of the earlier studies

\footnotetext{
1 The authors compare a constant-effort to a constant-catch rule under cyclical growth parameters. They briefly state that an optimal policy will be a pulse function, but do not provide analytical solutions to the optimal harvest rate under cyclical growth parameters.
} 
that incorporate randomness in the growth function by adding an i.i.d. error term, e.g., Reed (1979), Clark and Kirkwood (1986), Weitzman (2002), or Sethi et al. (2005). In the case of i.i.d. error terms the likelihood of observing several consecutive bad or good years is very small. Yet, cyclical growth functions with a long periodicity imply that several bad or good years are quiet likely.

We show that for periodically oscillating growth parameters, the harvest rate will also be cyclical, but more importantly, it lags the cycle of the growth parameters, i.e., the harvest rate peaks/bottoms after the biological growth parameters have peaked/bottomed. This result is contrary to the usual belief that harvest rates are monotonically linked to target stock levels. The time lag between the target stock and the harvest rate is important as existing regulations predominantly fix harvest rates (quantities) and not target stocks or escapement levels. Second, we examine under what conditions on the cyclical components harvest closures should occur and find that they do occur during periods when the growth parameters are improving most rapidly. Third, the standard management criteria of choosing the highest feasible time-invariant harvest quota (maximum sustainable yield, or MSY) can result in resource rents that are substantially lower than the economic optimum. ${ }^{2}$ Fourth, in a multi-species system, cycles with very moderate amplitude can lead to large and significant fluctuations in the optimal harvest rate of other fish species if the species are linked with one another.

There are only a few studies in the fisheries management literature that examine the role of cyclical growth parameters. The two studies closest to ours by biologists have received little attention by economists. In the first, Parma (1990) examines the case of systematically oscillating growth functions and the implications for optimal fisheries management for a risk neutral as well as a risk averse manager. Using a simulation approach, she finds that the optimal escapement (fish stock at the end of the period) fluctuates synchronously with the periodic growth parameter, i.e., escapement levels are raised when growing conditions are good and lowered when conditions are bad. The article focuses exclusively on stock-independent harvest cost, but allows for age-class models that incorporate time lags when fish can start to spawn. Because of the more elaborate age-structures, the paper relies on numerical simulations to derive optimal policies as the analytical solutions only give bounds. ${ }^{3}$ In the other, Walters and Parma (1996) consider periodic fluctuations potentially induced by climate change in an elaborate system of density-dependent and density-independent mortality rates, but the objective is to maximize total catch over a finite period rather than the resource rent from the fishery.

Papers in the economics literature in addition to Hannesson and Steinshamn (1991) that look at a potentially non-stationary growth rate include Costello et al. (1998), who look at

\footnotetext{
2 Biologists have hypothesized that it might be desirable to change the allowable catch in the case of fluctuating populations dynamics. For instance, Ricker (1975) first defines maximum sustainable yield as the largest average catch that could be taken from a stock under existing environmental conditions, but then parenthetically notes: "For species with fluctuating recruitment, the maximum might be obtained by taking fewer fish in some years than in others." Mangel et al. (2002) examine problems with the standard definition of maximum sustainable yield when there are large fluctuations in environmental conditions using the California commercial squid fishery as an example.

3 An unpublished paper from the mathematical population dynamic literature by Castilho and Srinivasu (2005) goes over some of the same ground as Parma (1990) with respect to the implications of a time varying growth rate for optimal management. There are many population dynamic models that have growth rates that are predictable functions of identifiable factors such as environmental conditions (Mallet et al. 1999). Singh et al. (2006), using the Pacific Halibut fishery as an example, is one of the few papers to introduce autocorrelated error terms in a model explicitly looking at management implications. This is relevant because one might expect to see autocorrelated error terms if the underlying periodic growth parameters were not adequately modeled. Empirical papers focused solely on modeling population dynamics with autocorrelated errors are reasonably common, e.g., Pyper and Peterman (1998).
} 
the value of El Nino forecasts on managing salmon stocks in the Pacific Northwest, and Costello et al. (2001), who look more generally at the role of predictable environmental conditions. ${ }^{4}$ Similar to Parma, Costello et al. (2001) show that a positive shock to the growth parameters will increase optimal escapement levels. They then go on to postulate that increasing escapement levels imply decreasing harvest rates (p. 207). While this might seem intuitive at first (higher escapement by definition implies that more fish have to remain in the sea and less can be harvested), recall that environmental conditions improved to begin with, and hence, more fish were available. We show that the harvest rate is out of phase with the cycle of the growth function, i.e., it is generally true that there are parts of the cycle where growth parameters are improving (i.e., fish growth is improving) and both the escapement level and the harvest rate are increasing at the same time. Since most fisheries regulation are in terms of harvest quantities (and not target stocks or escapement levels), the offset in the cycles of the optimal harvest quantity and the growth parameters is a policy-relevant extension that has not previously been recognized.

The rest of this paper is devoted to laying out a model that is consistent with the set of stylized facts put forth and examining its implications for fisheries management more thoroughly. In a first step we focus on the implications of a time-varying growth rate and carrying capacity for a single species. We start with a model in the spirit of Parma (1990) where harvest cost are independent of the fish stock to illustrate our main conclusions in Sect. 2. While previous authors show that the optimal target stock fluctuates synchronously with the biological growth parameters, we show that the optimal harvest rate is also cyclical but lags the cycle of the growth parameters, i.e., it peaks after the biological growth parameters already started to decline. It is best to close a fishery during times when the non-stationary biological growth parameters are improving most rapidly, and hence, the return from not fishing is highest. The same reasoning still holds when we introduce stock-dependent cost in Sect. 3. Section 4 compares the optimal harvest policy under cyclical fluctuations to traditional models of maximum sustainable yield and constant target stock/escapement levels, as well as adaptive rules where fishing quotas are repeatedly revised or set to a constant fraction of the fish stock. We show that a policy that derives the maximum sustainable harvest quantity using the average growth rate will lead to overfishing and collapsing fish stocks, as will adaptive policies that utilizes a limited time-series of past data.

Fluctuating growth rates may be one key aspect of moving towards an ecosystem based management system. Another is the interaction between species. In Sect. 5, we look at cyclically varying growth rates in the context of a predator-prey system with one predator species and two prey species. Small fluctuations in growth parameters in one species can lead to sizable fluctuations in optimal harvest policies of all species due to the interplay of the species. Finally, Sect. 6 concludes.

\section{A Motivating Example}

This section introduces cyclical fluctuations to a simple logistic single-species model with stock-independent harvest cost. The purpose of this section is to give an intuition for similar results we obtain in later sections.

\footnotetext{
4 Costello (2000) also considers the case of an an AR(1) error structure in his dissertation. In case of a positive auto-correlation coefficient, the fishery will experience prolonged periods of bad or good growth parameters. The value of utilizing the information on the AR(1) structure is demonstrated for the case of pink salmon in British Columbia.
} 
The unique feature of our setup is that there are predictable cycles in the biological growth process. This differs sharply from assuming i.i.d. error terms (Pindyck 1984). More specifically, consider a fish species with stock $F(t)$ at time $t$ that follows a logistics growth function $\dot{F}(t)=g(F)=\left[\alpha_{0}(t)+\alpha_{1}(t) F(t)\right] F(t)$ where $\alpha_{0}(t)>0, \alpha_{1}(t)<0$. Note that the growth rates $\alpha_{0}(t), \alpha_{1}(t)$ can be time-varying, and hence, so might be the carrying capacity $-\frac{\alpha_{0}(t)}{\alpha_{1}(t)} \cdot 5$ Assume that fisheries management maximizes the present value of future harvests $h(t)$.

As a first-step assume that there are constant marginal stock-independent harvesting cost $\omega$ and the price of fish is $P$. Using a discount rate $\delta(t)$ the optimization problem becomes ${ }^{6}$

$$
\max _{h(t)} \int_{0}^{\infty} e^{-\int_{0}^{t} \delta(s) d s}[p-\omega] h(t) d t \quad \text { s.t. } \dot{F}(t)=\left[\alpha_{0}(t)+\alpha_{1}(t) F(t)\right] F(t)-h(t)
$$

The optimal policy requires a target stock $F^{*}$ where the tangent to the growth function equals the interest rate. The intuition is quite simple: a fisherman faces the choice of either harvesting now and putting the proceeds in the bank to earn interest $\delta(t)$, or delaying harvest by some time to obtain some additional biological growth as given by the slope of the growth function. Since the slope of a logistic growth function is decreasing in the stock size, the biological growth exceeds the economic opportunity cost of earning interest rate $\delta(t)$ to the left of the stock level $F^{*}$, and is lower than the economic opportunity $\cos t \delta(t)$ to the right of it.

Proposition 1 The optimal harvest policy $h(t)$ is to keep the fish stock at the desired level $F^{*}(t)=\frac{\alpha_{0}(t)-\delta(t)}{-2 \alpha_{1}(t)}$ if feasible, or set the harvest rate to zero until the desired fish stock is reached.

The derivation is given in the appendix. Note the complete symmetry between fluctuations in the growth rate $\alpha_{0}(t)$ and the interest rate $\delta(t)$, as only the difference between the two determines the optimal stock size. Hence, business cycle models with cyclical interest rates will have analogous effects on the optimal harvest policy. ${ }^{7}$ In this paper we focus on cyclical biological growth parameters, but a similar story could be told for a stable time-invariant biological system when there are fluctuations in the interest rate.

In the remainder we fix $\delta$ and $\alpha_{1}$ but let $\alpha_{0}(t)$ be a synodal function. We assume that $\alpha_{0}(t)>\delta$ for all $t$, i.e., we rule out a border solution where it is best to harvest the entire stock of the fish species. Changing intrinsic growth rates translate into changing carrying capacities as well as changing optimal stock levels $F^{*}$ as shown in Fig. 1. Varying intrinsic growth rates $\alpha_{0}(t)$ imply a periodic carrying capacity $\frac{\alpha_{0}(t)}{-\alpha_{1}}$ as well as a periodic desired fish stock $\frac{\alpha_{0}(t)-\delta}{-2 \alpha_{1}}$, ranging from $F_{\text {min }}^{*}$ under the lowest intrinsic growth rate to $F_{\max }^{*}$ under the highest intrinsic growth rate. The optimal harvest policy is simply the difference between the growth rate and the change in the desired fish stock, i.e., $\frac{\alpha_{0}(t)^{2}-\delta^{2}-2 \alpha_{0}^{\prime}(t)}{-4 \alpha_{1}}$ (for a case where there are no harvest closures, i.e., the above fraction is never negative).

Intuitively, if the system is at $F_{\max }^{*}$, and the intrinsic growth rate $\alpha_{0}(t)$ starts to decline, the slope of the growth function at $F_{\max }^{*}$ becomes smaller than the interest rate, suggesting that the return of keeping an extra fish in the ocean is less than the opportunity cost of earning interest $\delta$. Hence, one should reallocate funds to the investment opportunity with the larger

\footnotetext{
5 Reconstructions of historical fish stocks suggest that the carrying capacity of many fish stocks used to be much larger (Jackson et al. 2001).

6 We assume $\alpha_{0}(t)>\delta(t)$, so it is never optimal to wipe out the fishery.

7 Abstracting from time-inconsistency problems that might arise due to changing interest rates.
} 


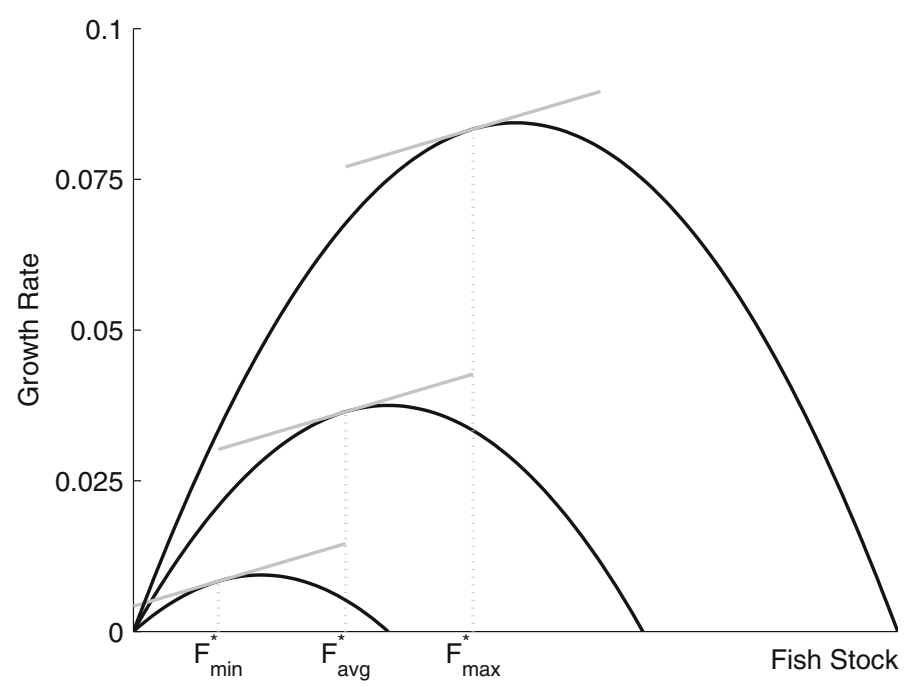

Fig. 1 Oscillating intrinsic growth rate with stock-independent harvest cost. Notes: The graph displays the time-varying growth function and optimal stock levels $F^{*}$ fluctuating between $F_{\min }^{*}$ and $F_{\max }^{*}$. The parameter values are $\alpha_{0}=0.15+0.075 \sin \left(\frac{2 \pi t}{50}\right), \alpha_{1}=-0.15, \delta=0.025$

return, i.e., decrease the fish stock, thereby raising its return until it equals the opportunity cost again. The opposite is true as well: if the system is at $F_{\min }^{*}$ and the intrinsic growth rate $\alpha_{0}(t)$ starts to rise again, the slope of the growth function at $F_{\min }^{*}$ becomes larger than the interest rate, suggesting that the additional return of keeping an extra fish in the ocean exceeds the opportunity cost of putting the proceeds from harvesting fish in the bank and earning interest. Thus, one should "invest" into the fish stock, i.e., shift resources into the fishery up to the point where its return equals the market return again. Hence, in a deterministic model of cyclical population dynamics, harvest rates (e.g., individual transferable quotas) should not be specified as a constant catch per period but should themselves be time-varying.

There is one asymmetry in the model: one can draw down the fish stock instantaneously to any arbitrary nonnegative amount and place the proceeds from selling the fish into an interest bearing bank account, but the opposite is not true: the natural growth rate places an upper bound on the increase in the fish stock as negative harvest rates are infeasible. Hence, if the natural growth rate of the fish population does not diminish the return on fishing enough to decrease it to $\delta$, it is best to close the fishery to harvest.

The intuition for a harvest closure are very similar to the standard case of a stationary growth function: the manager has to ensure that the slope of the growth function equals the interest rate. In the traditional model, if a random shock decreases the fish stock below its optimal level, it is best to prohibit harvesting. Similarly, positive shocks to the fish stock warrant increased harvest rates to draw down the fish stock to the point where the slope of the growth function equals the opportunity cost of earning interest $\delta$. This bang-bang solution is driven by the linearity of the value function for two investment opportunities: (i) fisheries and (ii) the opportunity cost of earning interest $\delta$. Arbitrage requires that the returns of the two are equal. The difference in our model is that we are not examining shocks to the fish stock but rather introduce a cyclical growth function that is changing with time. Traditionally, increasing fish stocks imply that the return on fishing is below the interest rate (due to the concavity of the growth function). In our model, increasing fish stocks signal that the growth 
rate itself is increasing and that the returns on fish growth exceed the interest rate. The system is entering a period with the most rapid biological growth and it might pay to close the fishery. In our case of predictable fluctuations, harvesting is prohibited not because the fish species experienced a negative shock, but because it is entering a positive recovery phase with exceptionally good growth rates, and hence, the return on not harvesting is particularly large. Thus, harvest closures only happen during upswings, i.e., when growth conditions improve!

In the remainder of this paper we use the following specification of the time-varying synodal intrinsic growth rate: $\alpha_{0}(t)=\alpha_{01}+\alpha_{02} * \sin \left(\frac{2 \pi\left(t+\alpha_{03}\right)}{\alpha_{04}}\right)$, i.e, $\alpha_{01}$ is the average intrinsic growth rate, $\alpha_{02}$ the amplitude of the cyclical fluctuations, $\alpha_{03}$ an offset that allows the start of the cycle to be shifted in time, and $\alpha_{04}$ the periodicity. As mentioned above, the harvest rate becomes zero when the growth rate $\left[\alpha_{0}(t)+\alpha_{1} F^{*}(t)\right] F^{*}(t)$ is less than the growth in the desired stock level $\dot{F}^{*}(t)=\frac{\alpha_{0}^{\prime}(t)}{-2 \alpha_{1}}$, i.e., the change in the optimal stock size outpaces the natural growth rate. The following proposition establishes that there exists a minimum amplitude for which harvest closures become optimal and how it relates to the average intrinsic growth rate and periodicity.

Proposition 2 The minimum amplitude $\alpha_{02}$ for which there is a harvest closure in the cyclic single-species model is increasing in the intrinsic growth rate $\alpha_{01}$ and periodicity $\alpha_{04}$, and decreasing in the interest rate $\delta$.

The proof is given in the appendix. Intuitively, a larger periodicity implies that the desired change in fish stock happens fairly smoothly and can be sustained by natural growth. If on the other hand the desired fish stock changes very rapidly, the desired increase may not be achieved by natural fish growth and it is optimal to set the harvest rate to zero. Accordingly, if the amplitude is $\alpha_{02}$ too large (and the desired fish stock has a larger amplitude as well), the desired changes in fish stock cannot be sustained. In the extreme, once the amplitude $\alpha_{02}$ approaches the difference between the average growth rate $\alpha_{01}$ and the interest rate $\delta$, the minimum desired fish stock approaches zero, at which point the fish growth approaches zero as well. Once the biological parameters improve again, the desired fish stock increases, but this increase cannot be sustained because the natural growth is too low. The results for a particular set of parameter assumptions are shown in Fig. 2.

Note that the fish stock $F$ can drop below the desired stock level $F^{*}$. One might wonder whether a zero harvest rate and a stock level below the desired stock level $F^{*}$ can be optimal. Would it not be preferable to reduce the harvest rate at an earlier time when it is still positive to avoid the predictable harvest closure? Again, the harvest closure occurs not because the fish stock is in a "bad" condition but because the growth rate at the current stock size is increasing sufficiently fast. Reducing harvest at an earlier point would imply an investment in a bad state (when the growth rate is even lower) in order to increase harvesting in a better state (a time period when the growth rate is larger). This cannot be optimal, as investing in an asset should not occur during periods of low growth but during periods of high growth.

Proposition 3 The desired fish stock $F^{*}(t)$ fluctuates synchronously with the biological growth parameter $\alpha_{0}(t)$, but the cycle of the optimal harvest rate lags the cycle of the biological growth parameter.

The proof is given in the appendix. The result that the desired fish stock varies synchronously with the biological growth parameters is immediately evident from the solution of the desired fish stock $F^{*}(t)=\frac{\alpha_{0}(t)-\delta}{-2 \alpha_{1}}$ in Proposition 1. The intuition behind why the optimal harvest rate lags behind the desired fish stock is as follows: the optimal harvest rate is 


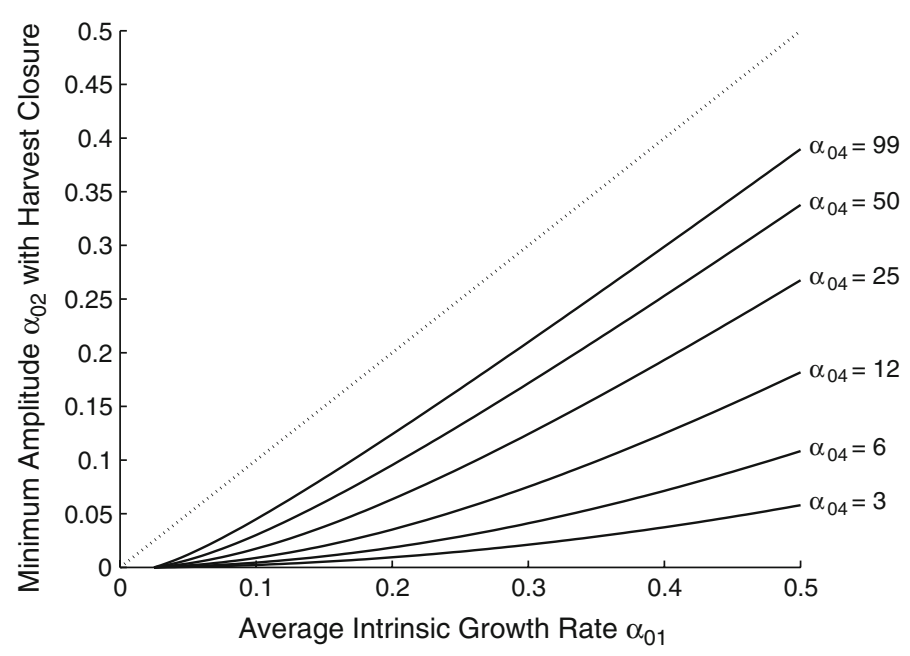

Fig. 2 Oscillating intrinsic growth rate with stock-independent harvest cost. Notes: The graph displays the minimum amplitude that implies a harvest closure as a function of mean intrinsic growth rate $\alpha_{01}$ and periodicity $\alpha_{04}$. The parameter values are $\alpha_{0}=0.15+0.075 \sin \left(\frac{2 \pi t}{50}\right), \alpha_{1}=-0.15, \delta=0.025$

the difference between the growth rate and the change in the optimal stock level. The time derivative of the former is zero at the same time the biological growth parameter $\alpha_{0}(t)$ peaks, however the time derivative of the latter includes the change of the change in the biological growth parameter, which is negative when the synodal growth parameter is largest (as the sin curve is concave on $[0, \pi]$ ) and positive when the synodal growth parameter is smallest (as the sin curve is convex on $[\pi, 2 \pi]$ ). In other words, the change in the change of the optimal stock level incorporates that one is switching from a period of increasing desired fish stocks to a period of decreasing fish stocks (or vice versa) giving an extra incentive (disincentive) to fish as the stock will decrease (increase).

This result has important policy implications as it implies that optimal harvest rates (e.g., transferable quotas) should be out of phase with the cycle in the biological growth parameters. To our knowledge this implication has not been previously noted.

Some disclaimers of our model are in order: Our setup maximizes the discounted profits. Prices are assumed to be independent of harvest quantity (which appears reasonable for fisheries that constitute a small overall market share). If a regulator wanted to keep employed labor constant, there is a countervailing incentive to smooth fluctuations over time. One setup that has previously been used is to instead maximize the $\log$ of discounted profits (Parma 1990). The log of profits gives an incentive to smooth the variability as outlined in the extension to Proposition 1 in the appendix. Similar results hold for the following sections, i.e., if the regulator is risk-averse or has policy objectives that dislike fluctuations, the chosen policy will tend to smooth out the predicted cycles. In the extreme, a policy that requires time-consistency (i.e., constant harvest), will equal the maximum sustainable yield.

\section{Stock-Dependent Harvest Cost—Single Species}

The previous section introduced the case of stock-independent harvest cost to motivate why the periodic fluctuations in the harvest rate lag the cycle of the biological growth function 
and why harvest closures will occur during periods of increasing growth rates. We will now show that both results hold for the case of stock-dependent harvest cost as well. Authors as far back as Scott (1955) have recognized that if fishing cost depend on the fish stock, there might be an incentive to cut back fishing today for lowered fishing costs tomorrow. In the remainder of this section we rely on the standard modelling framework where harvest $h(t)$ is a linear function of effort $e(t)$ and stock size $F(t)$, i.e., $h(t)=\theta e(t) F(t){ }^{8}$ Furthermore, there is a constant marginal cost of effort $\omega$ as well as constant fish price $p$. The regulator is maximizing the discounted value of future profits with discount rate $\delta$, i.e., the problem becomes

$$
\max _{h(t)} \int_{0}^{\infty} e^{-\delta t}\left[p h(t)-\omega \frac{h(t)}{\theta F(t)}\right] d t \quad \text { s.t. } \quad \dot{F}(t)=\left[\alpha_{0}(t)+\alpha_{1} F(t)\right] F(t)-h(t)
$$

The optimal solution requires that the stock of the fish species is kept at $F^{*}$, which is itself a cyclical function of $\alpha_{0}$.

Proposition 4 The optimal harvest policy $h(t)$ is to keep the fish stock at the desired level $F^{*}(t)=\frac{\theta p\left[\alpha_{0}(t)-\delta\right]-\alpha_{1} \omega}{-4 \alpha_{1} \theta p}+\sqrt{\left(\frac{\theta p\left[\alpha_{0}(t)-\delta\right]-\alpha_{1} \omega}{-4 \alpha_{1} \theta p}\right)^{2}+\frac{\delta \omega}{-2 \alpha_{1} \theta p}}$ iffeasible, or set the harvest rate to zero until the desired fish stock is reached.

The derivation is given in the appendix. ${ }^{9}$ The formula in Proposition 4 is the solution for the dynamic optimum implied by $g^{\prime}\left(F^{*}\right)+\frac{\omega g\left(F^{*}\right)}{F^{*}\left(\theta p F^{*}-\omega\right)}=\delta$ where $g(F)=$ $\left[\alpha_{0}(t)+\alpha_{1} F(t)\right] F(t)$ is the logistic growth function. It differs from the solution in the standard static framework of the previous subsection where the slope of the logistic growth function equals the interest rate, $g^{\prime}\left(F^{*}\right)=\delta$. In the static setting the equilibrium is determined where the marginal growth rate equals the discount rate, i.e., the point where the return on holding natural resources is equivalent to the market return. The dynamic optimum incorporates the fact that a change in current harvest also influences the profit margin in the future, as harvesting costs are inversely proportional to the stock size. This effect is captured in the term $\frac{\omega g\left(F^{*}\right)}{F^{*}\left(\theta p F^{*}-\omega\right)} \cdot{ }^{10}$ The solution for the case of stock-independent harvest cost in Proposition 1 is just a special case of Proposition 4 where $\omega=0$. Standard comparative static results for the case of stock-dependent harvest cost are given in the following proposition.

Proposition 5 The desired stock level is increasing in the growth parameters $\alpha_{0}$ and cost of effort $\omega$, while it is decreasing in the growth parameter $-\alpha_{1}$, effort factor $\theta$ and price offish $p$.

The comparative static results are given in the appendix, and we focus on the intuition behind these results: a larger intrinsic growth rate $\alpha_{0}$ implies larger biological growth which is more conducive to support larger fish stocks. On the other hand, $-\alpha_{1}>0$ measures the crowding out effect of the fish species, which implies that a smaller fish stock can be supported. A higher price for fish implies that the tradeoff of fishing now or keeping a higher

\footnotetext{
8 The model assumes that the amount of fish caught per unit of effort is proportional to the fish stock.

9 Our objective function assumes a constant price of fish and cost of effort. It does not include adjustment cost or risk aversion that might induce a regulator to smooth profits between periods. Moreover, the behavioral responses of fishing fleets can have important implications. One might expect two reactions to cyclical growth parameters. The first is that adjustment cost might dampen out the cycle. The second is that fleets would re-equip to be able to harvest other fish species which might have the opposite effect.

10 Note that the symmetry between a cyclical interest rate $\delta$ and a cyclical growth rate $\alpha_{0}$ no longer holds with stock-dependent harvest cost. This is due to the fact that biological growth parameters impact the fishing cost in the next period, while the interest rate does not.
} 
stock to lower future fishing cost is further tilted towards fishing now. Higher harvest cost $\omega$ implies that it is optimal to keep a larger fish stock to lower future harvest cost. As $\omega$ approaches zero, the solution converges from above to the one with stock-independent cost. Therefore, the desired fish stock is always higher for the case of stock-dependent harvest cost. Finally, an increase in $\theta$, the efficiency factor on fishing effort is equivalent to a decrease in the cost of fishing $\omega$ thereby decreasing the desired fish stock.

Since the intrinsic growth function $\alpha_{0}$ is fluctuating, so is the desired optimal stock level $F^{*}(t)$ and the harvest rate $h(t)$. The following proposition establishes that the cycle of the optimal harvest policy always lags behind the cycle of the biological growth function, i.e., the harvest rate peaks once the biological growth function has started to decline, and bottoms out once the biological growth function has started to increase.

Proposition 6 The desired fish stock $F^{*}(t)$ varies synchronously with the periodic biological growth function, while the cycle of the harvest policy $h(t)$ lags behind the cycle of the biological growth function.

The proof is given in the appendix. The intuition behind this result is the same as under stock-independent harvest cost: the desired stock level $F^{*}(t)$ varies synchronously with the cyclical biological growth function, i.e., it peaks at the same time. The optimal harvest rate is the difference between the growth rate and the change in the optimal stock level. The change in the harvest rate therefore depends on the change in the change of the optimal stock level, which includes the second derivative of the growth function. If the synodal growth function peaks, this second derivative is negative, and the desired harvest rate is still increasing. In other words, once the growth function peaks, the regulator realizes that the investment opportunity of leaving fish in the sea becomes less profitable in the future, and so it is optimal to decrease the desired stock level or further increase the harvest rate. The same logic holds when the growth function is at its minimum; the regulator realizes that the system is now entering the phase with the highest return, so it pays to keep more fish in the sea and further decrease the harvest.

The same intuition will hold if we were to relax other model assumptions, e.g., allow for convex harvest cost. While convex harvest cost give a disincentive to vary harvest rates over time and would smooth some of the optimal fluctuations, the peak level will still lag behind the optimal growth function for the reasons given above. Similarly, the preceding analysis has focused on the case of cycles with constant periodicity. Real-world fisheries will exhibit growth parameters that fluctuate with a periodicity of random length. For example, the time between consecutive El Nino years is random. One might wonder what would happen to the optimal harvest solution in this case. To illustrate this point consider two extremes: if the periodicity is completely predictable and certain, the desired fish stock is cyclical as well. It fluctuates synchronously with the biological growth parameters. On the other hand, if the growth parameters were constant and all fluctuations were caused by random shocks, the optimal policy is given by a time-invariant fish stock. Now if we were to consider a hybrid where the random periodicity in a growth parameter is overlaid with random shocks, the optimal solution will be a hybrid, i.e., the desired fish stock will fluctuate but with an amplitude that decreases in the overlaid variance of the randomness. Intuitively, the more uncertainty there is in the periodicity of the growth function, the more the optimal solution again approaches the time-invariant fish stock. The applicability of the results depend on how well the cycles of the underlying growth parameters are understood and how well they can be estimated.

The next section contrasts the optimal time-variant policy from traditional fishing policies with the help of simulations. While we have shown formally in the last two sections that the 
optimal policy will differ, one might wonder how large the welfare losses are from using one of the suboptimal policies.

\section{Simulation of Various Harvest Policies}

We can now use the analytical results of the previous section to simulate economic rents and fish stocks under the optimal harvest rule and compare it to various stylized fishing policies underlying fishing regulation.

A traditional form of regulation is a government-imposed limit on the harvest quantity or harvest time. A maximum sustainable yield is the largest time-invariant constant harvest quantity that can be achieved forever.

Economists have often advocated a constant target stock in the past. The advantage of such a policy over one with constant harvest quantities is that the former deals better with random shocks to the system. It gives rise to a bang-bang solution, where increases in fish stock will immediately be offset by increases in harvest quantities, and declining fish stocks will lead to harvest closures. In reality, fishery counsels sometimes set the total allowable catch, and individual fishery quotas allow the owner to a pre-specified fraction of this catch.

Other policies we consider are a harvest quantity that is based on the average growth rate and an adaptive policy where the harvest quantity is periodically updated. In many cases regulators deliberately rely only on a limited historic record of fish stocks with the idea that it is more representative of the current fishery system. It should be immediately clear that such a policy will give an inaccurate assessment in a cyclical population model with a sufficiently long periodicity, as too large (small) maximum allowable harvest quantities are set when the system is entering the low (high) growth phase.

Finally, we simulate how well a regulator could estimate the periodicity from past observations. There is an initial period where it is difficult to distinguish random shocks from cyclical movements, but within a few years the regulator may be able to get reasonably precise estimates of the system.

In the remainder, the optimal harvest rule will be such that $\dot{F}(t)-h(t)$ keeps $F(t)$ at $F^{*}(t)$ if possible, otherwise $h(t)$ will be zero. We assume that the unit price of effort is $\omega=2$, the multiplicative factor on effort is $\theta=1$, the price of fish is $p=225$, the discount rate $\delta=0.025$, and the growth parameters are $\alpha_{0}=0.15+0.075 \sin \left(\frac{2 \pi t}{50}\right) ; \alpha_{1}=-0.15$, i.e., the carrying capacity is on average one. ${ }^{11}$ The system is started in a state without human intervention; and, we evaluate both the economically optimal policy as well as the traditional policies for each of the 50 starting points in the assumed 50-year periodicity.

\subsection{Maximum Sustainable Yield (Harvest Quantity)}

One of the most widely used concepts in the fishery literature is that of a maximum sustainable yield, i.e., the highest time-invariant harvest rate that can be sustained forever. Figure 3 displays the time-invariant maximum sustainable yield in black as well as optimal harvest policy in grey. The starting value in the figure is set to equal the fish stock without human intervention.

11 Both the average growth parameters and the economic parameters are taken from Perman et al. (2003), a standard graduate natural resource economics text. The periodicity of 50 years is taken out of Taylor (2004), who looks at long term climate change and salmon populations. 

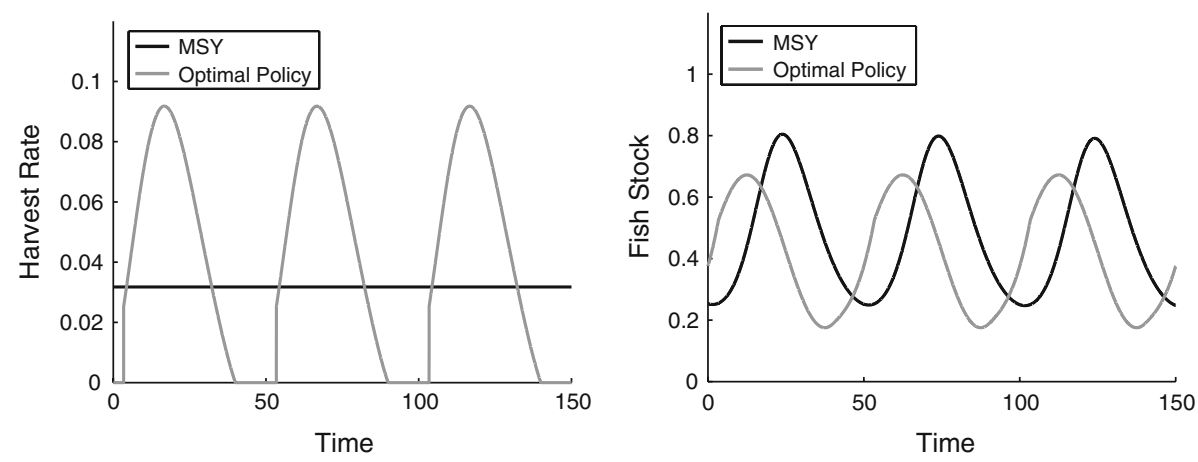

Fig. 3 Maximum sustainable yield under oscillating growth rates. Notes: The left panel displays harvest rates while the right panel shows resulting fish stocks. The black line uses the maximum sustainable time-invariant harvest rate while the grey line uses the optimal policy. The parameter values are $\omega=2, \theta=1, p=225, \delta=$ $0.025, \alpha_{0}=0.15+0.075 \sin \left(\frac{2 \pi t}{50}\right), \alpha_{1}=-0.15$

Note that the harvest rate under the optimal policy fluctuates significantly over time and sometimes even equals zero. A harvest moratorium occurs during time periods when the biological growth parameters rapidly improve and the return on not fishing is highest. The desired fish stock $F^{*}$ increases faster than what can be sustained by natural fish growth.

The optimal time-varying harvest rule results in substantially larger discounted net benefits. ${ }^{12}$ Under the optimal harvest rate, the average discounted stream of profits equals 493 , ranging from 423 under the least favorable of the 50 possible starting conditions to 573 under the most favorable starting condition. For the maximum sustainable yield the average discounted profits decrease to 282 .

The maximum sustainable harvest rate, by definition, is time-invariant. The highest sustainable rate in our system is 0.0317 , which is lower than the mean harvest rate under the optimal harvest policy, which is 0.0417 . It should be noted that the maximum sustainable yield in a standard stationary model is at least as high as the economically optimal harvest rate, as future harvest is traded for current harvest which is discounted at a lower rate. However, under the cyclical model this fact is no longer true. By forcing the harvest rate to be time-invariant, the constant harvest rate is limited by the phase of lower growth. Also, the maximum sustainable yield is lower than the one attainable under the average growth rate, which is $\frac{\alpha_{01}^{2}}{-4 \alpha_{1}}=0.0375$.

A time-invariant harvest rate together with fluctuating growth parameters imply that the fish stock is fluctuating over time as shown in the right graph of Fig. 3. The stock level under the constant harvest rate lags the cycle of the optimal stock level, while the latter varies synchronously with the biological growth function.

\subsection{Constant Target Stock}

The economic optimum in the standard stable continuous-time fisheries model implies that the fish stock should be kept at a constant level. The result holds even in the presence of random shocks in a discrete time setup, where the target stock is usually called escapement level.

\footnotetext{
12 The optimality of the time varying rules owes much to assumption that price is constant and costs are variable. These assumptions should be examined in future work if environmental conditions are broad enough to impact the price of fish or influence the pattern of investment.
} 

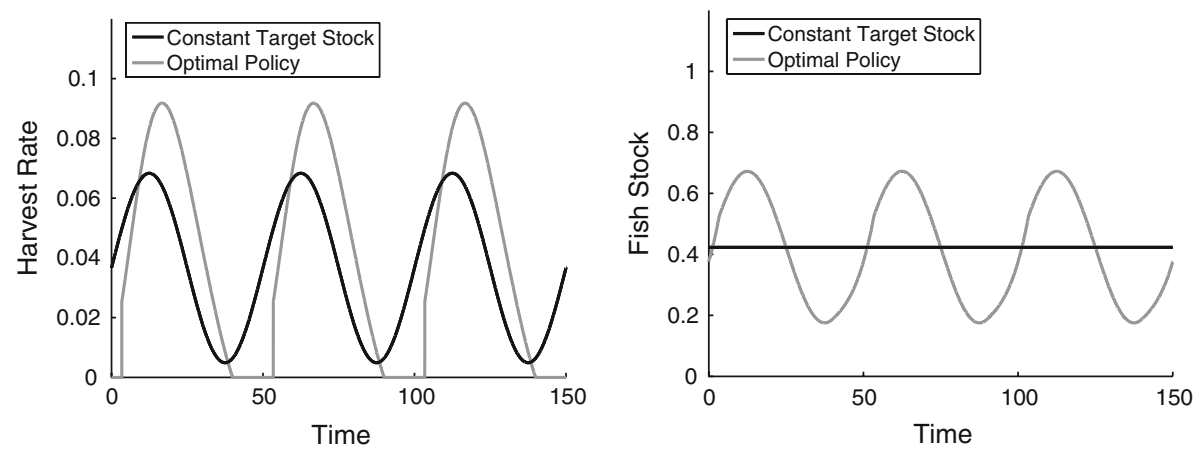

Fig. 4 Constant target stock under oscillating growth rates. Notes: The left panel displays harvest rates while the right panel shows resulting fish stocks. The black line uses a constant target stock while the grey line uses the optimal policy. The parameter values are the same as in Fig. 3

In the following we examine how well such a constant target stock fares in light of cyclical population dynamics. We use the same cyclical changes in the growth function as in the preceding section, yet assume the regulator relies on the average growth rate when designing its regulation. ${ }^{13}$ Figure 4 displays the resulting harvest rate and fish stock under such a scenario where the regulator incorrectly assumes that there is a stable time-invariant logistic growth function with $\alpha_{0}=0.15$. The harvest policy now peaks at the same time as the biological growth parameters, yet, as shown in previous sections, the optimal harvest policy lags behind, i.e., peaks later, as shown in the left graph of Fig. 4. While the maximum sustainable yield by definition holds the harvest quantity unchanged, the constant target stock fixes the stock size, even though the latter should fluctuate under the optimum. The biggest problem is that the harvest rate is too low during good, i.e., highly productive, times. Using a time-invariant target stock results in an average net benefit (over the 50 possible starting conditions) of 451 , which is inferior to the optimal time-variant policy of 493.

Weitzman (2002) shows that landing fees are preferable to harvest quantities in a stochastic setting with i.i.d. error terms as they generate the same solution as the constant escapement rule. However, under cyclical population dynamics, the landing fees would have to be cyclical since the optimal target stock/escapement level fluctuates. While many previous studies allowed for random shocks in the system, the large majority assumed that the logistic growth function is time-invariant or stationary. Similar to an error correction model in macroeconomics, the optimal harvest rate is not a stationary function but varies with the underlying growth parameters. ${ }^{14}$

\subsection{Quantity Regulation Based on the Average Growth Rate}

Harvest quantities are sometimes preferred over fees or constant target stocks as they are easier to implement and give fishermen certainty about the allowable catch in the next period. In the following we therefore consider two policies that use the average intrinsic growth rate in the design of the harvest quantity.

\footnotetext{
13 This section is designed to compare a constant target stock under the standard setup (that does not account for time-varying parameters) to the optimal time-varying one. There might be a constant target stock that gives higher average profits than the one based on average parameters, however, if a regulator were to realize that the growth function is cyclical, it is unclear why he/she would opt for a suboptimal constant instead of a time-varying target stock.

14 As an alternative to modeling a time-variant process, one could use time as a state variable itself.
} 

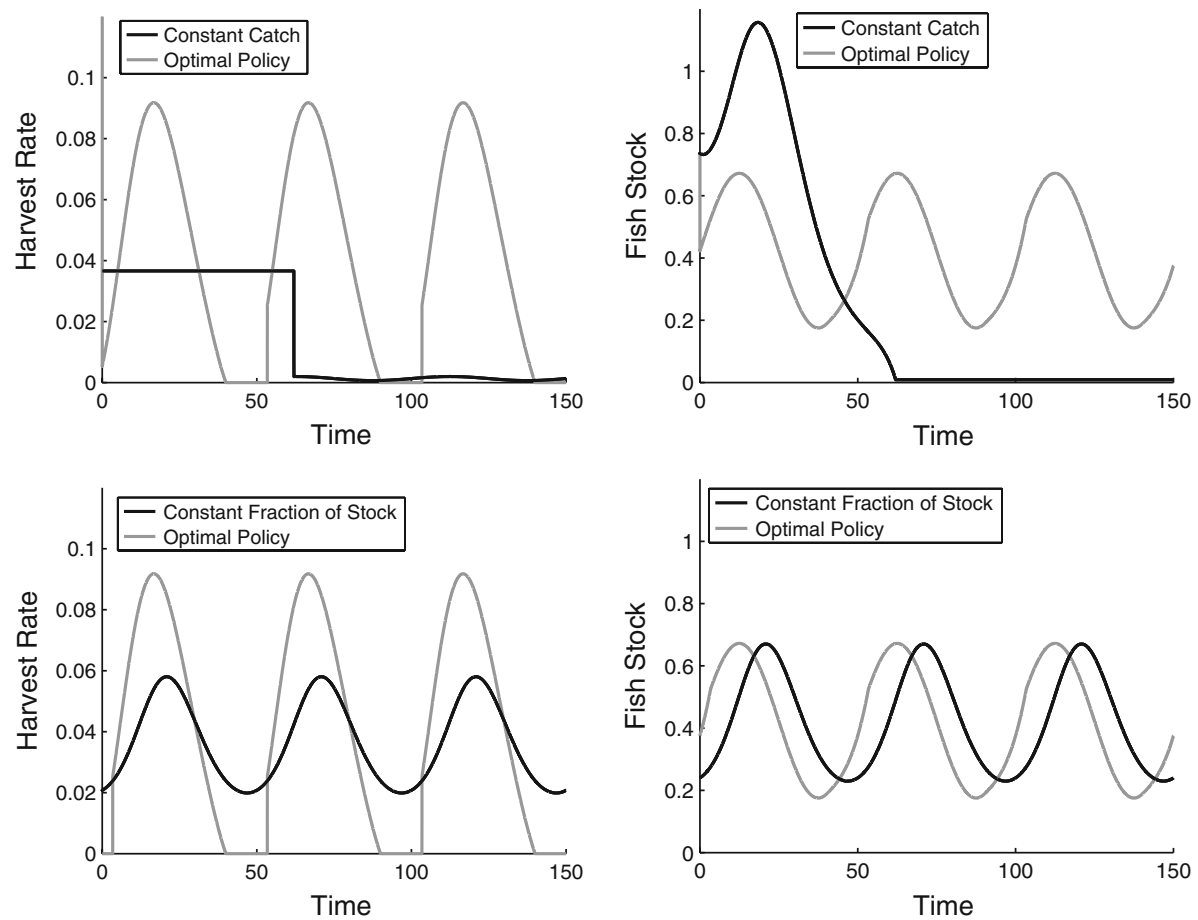

Fig. 5 Harvest rules based on average growth rates. Notes: The left panels display the harvest rates while the right panels show the resulting fish stocks. The top row uses the optimal catch (quantity) under the average growth rate. In the bottom row a constant fraction of the stock is harvested. The fraction is set to equal the ratio of the optimal harvest to fish stock under the average growth rate. The optimal policy is added in grey. The parameter values are the same as in Fig. 3

Results for the case where the regulator sets the harvest rate equal to the growth rate at the optimal stock under the average growth function are shown in the top row of Fig. 5. There are some interesting policy implications: First, the stock level diminishes to the open-access level, as the average harvest rate is too high. ${ }^{15}$ This is in line with the argument by Roughgarden and Smith (1996) that the optimal harvest level is not ecologically sustainable. Once the stock falls below it, continued use of the same harvest rate will further diminish the stock and harvest rate. Their recommendation was to keep the stock above the level associated with the maximum sustainable yield. Second, the harvesting rate will sometimes be lower than the allowable harvest rate, i.e., the regulation is not binding. Once the stock size drops to the open-access equilibrium, further fishing becomes unprofitable. ${ }^{16}$ The average discounted profits accordingly decline to 251 .

While a constant harvest rate (quantity) is not ecologically sustainable, setting the allowable catch equal to a constant fraction of the stock size is sustainable as deteriorating conditions result in lower harvest rates. Walters and Parma (1996) emphasize that a constant exploitation rate is better at adjusting to changing conditions as expected under climate change. While the policy does not result in the highest average harvest, the results are gen-

\footnotetext{
${ }^{15}$ It is of course possible to have cases where the periodic fluctuations are large enough to lead to short-term recoveries where the fishing quota becomes binding again.

16 We have assumed a constant price of fish. There is a critical stock level where further fishing results in negative returns (the catch per unit of effort is low enough).
} 
erally within $15 \%$ in their simulations. A key advantage of such a policy is that it does not require any forecast of predicted biological conditions in the next period, as it is solely based on the observed fish stock in the current period. For cyclical growth parameters, the results of such a policy are displayed in the second row of Fig. 5 as a black line. The optimal harvest policy is included as a grey line for reference.

We set the constant fraction of the stock that is harvested to equal the ratio of the optimal catch to the stock size under the average growth function. This policy partially simulates a system of fluctuating harvest rates, as periodic fluctuations in growth parameters translate into fluctuating stock sizes and harvest rates. However, these fluctuations are of lower magnitude and offset compared to the optimum policy. The average discounted profits are 411 compared to 493 under the optimum.

\subsection{Adaptive Policy}

In this section we allow for an adaptive policy where the regulator realizes that the underlying system is changing and repeatedly updates his or her estimate for the growth parameters. The motivation of such a policy is that US regulations sometimes require periodic review of harvest quotas and that older data is often considered suspect from a quality or relevancy perspective.

Specifically, we consider the case of a regulator who re-estimates the logistic growth function every 5 years using data on fish stocks and harvest from the previous two decades. The regulator again assumes that the carrying capacity is fixed, and only estimates the instantaneous growth function from the discretized version $h_{t}+F_{t+1}-F_{t}=\alpha_{0}\left[1-F_{t}\right] F_{t}+\epsilon_{t}$. We include an error term in this subsection as the regulator is estimating a model from observed data. Without random shocks, the model can be solved analytically. The random shocks simply give us added variation in the stock, but we assume the regulator immediately observes these shocks. The results from our analytical section still apply here, and hence the goal is to keep the fish stock at the derived optimal target stock that varies over time, immediately offsetting any shocks to the system.

We simulate a stochastic system using a standard Wiener process $W(t)$ with $d F(t)=$ $\left[\alpha_{0}+\alpha_{1} F(t)\right] F(t)+\sigma F(t) d W(t)$ and aggregate the observations on a yearly basis. The logistic growth parameter $\alpha_{0}$ is estimated from the 20-year history preceding each policy re-evaluation. In case the current stock of fish is above the optimal stock level, the harvest rate is set equal to the growth rate at the optimal stock level. In case the stock of fish is below the optimal stock level, the fishery is closed until it has reached the optimal stock level again, upon which the harvest rate is set to equal the growth rate at the optimal stock level. The resulting harvest rates and stock levels under both the optimal policy and the one using lagged values is displayed in Fig. 6. We switch the periodicity from 50 to 49 years to avoid it being a multiple of the reauthorization process of 5 years.

Note how the adaptive policy repeatedly sets the allowable harvest rate too high when growth rates are declining. These fishing limits turn out to be too high during periods of slow growth rates and result in serious overfishing. The fish species is saved from complete extinction as fishing efforts are stopped as soon as the population reaches the open access equilibrium and further fishing becomes unprofitable. ${ }^{17}$ Once the regulation is re-evaluated, the fishery has to be closed until the stock has recovered.

17 We purposefully pick a low marginal cost of effort in comparison to the price of fish. This seems appropriate as the largest share of total cost is usually fixed cost, especially for the highly capitalized modern fleet that captures the largest fraction of fish. 

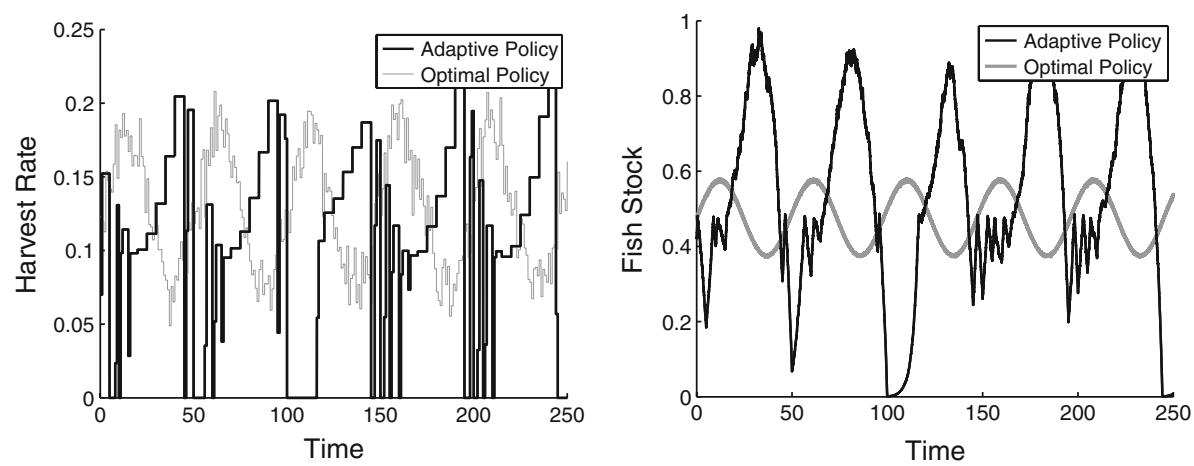

Fig. 6 Lagged government policy. Notes: The left panel displays the harvest rates, while the right panel shows the resulting fish stocks. The black line uses a lagged policy where the regulator is assumed to estimate the instantaneous growth rate $\alpha_{0}$ using the 20-year history preceding each policy reauthorization (which occurs every five years); and sets the harvest quota equal to the growth rate at the optimal stock level for the next 5 years in case the stock level is above the optimum stock level, and closes the fishery otherwise. The grey line displays the optimal harvest rate (averaged over each year). The parameter values are $\omega=0.5, \theta=1, p=$ $500, \delta=0.025, \alpha_{0}=0.5+0.1 \sin \left(\frac{2 \pi t}{49}\right), \alpha_{1}=-0.5, \sigma=0.03$

The repeated over-fishing, closure, and re-opening leads to significantly lower average net benefits. The problem becomes worse if we assume that private companies invest in more capital-intensive boats with lower marginal cost during good periods, which in turn yields a lower open-access equilibrium $\frac{\omega}{\theta p}$ during times of decreasing biological growth parameters when the fishing limits based on lagged data are too high. Recall that the lower stock level in the right graph of Fig. 6 equals the open-access case where it is no longer profitable to fish. Once the marginal costs are very low, there is a real danger that the fish species will not recover, especially if there were to be a minimum sustainable level below which the species cannot recover.

\subsection{Moving to a New Management Regime}

Table 1 summarizes the advantages and disadvantages of various harvest policies we have discussed. If cyclical population dynamics are significant for a given fishery species, how would a regulator best implement a new management regime? Initially, the fishery will almost certainly be off any desirable equilibrium path. The optimal response under our model setup would be a bang-bang solution to adjust to the equilibrium path as soon as possible. However, many fisheries around the world are managed with additional objectives besides rent maximization, e.g., maintaining a stable employment in the fishing industry. This will generally rule out a bang-bang solution that calls for a fishery closure until the stock has recovered to desired levels. Moreover, as noted above, cyclical population dynamics calls for time-variant harvest rates that lead to swings in employment (assuming constant fishing technology). This forces the regulator to balance two countervailing objectives: (i) rent maximization and (ii) stable employment. While there is political pressure for stable employment, the latter only allows for lower employment than can be realized on average under the former.

\subsection{Estimating Periodic Growth Parameters}

So far we have always compared the optimal policy to suboptimal time-invariant or lagged policies. Recognizing the inferiority of the time-invariant policies in terms of discounted 
Table 1 Comparing various harvest policies

\begin{tabular}{|c|c|c|}
\hline Policy & Pros & Cons \\
\hline "Optimal" policy & Highest profit & $\begin{array}{l}\text { Requires accurate forecast of biological } \\
\text { cycle }\end{array}$ \\
\hline MSY & $\begin{array}{l}\text { Constant harvest rate (if } \\
\text { regulator dislikes } \\
\text { cyclical outcomes) }\end{array}$ & $\begin{array}{l}\text { No adjustment if fish stock drops below } \\
\text { critical level and fishery might } \\
\text { collapse. Lower profits }\end{array}$ \\
\hline Constant target stock & $\begin{array}{l}\text { Automatic adjustment to } \\
\text { changes in fish stock } \\
\text { Does not require a } \\
\text { forecast of future } \\
\text { conditions }\end{array}$ & Lower profits \\
\hline $\begin{array}{l}\text { Constant catch (average } \\
\text { growth rate) }\end{array}$ & & $\begin{array}{l}\text { Fisheries collapse if optimal catch is } \\
\text { based on average growth rate }\end{array}$ \\
\hline \multirow[t]{2}{*}{$\begin{array}{l}\text { Constant fraction } \\
\text { of stock (average } \\
\text { growth rate) }\end{array}$} & $\begin{array}{l}\text { Automatic adjustment to } \\
\text { changes in fish stock }\end{array}$ & Lower profits \\
\hline & $\begin{array}{l}\text { Does not require a } \\
\text { forecast of future } \\
\text { conditions }\end{array}$ & \\
\hline Adaptive policy & & $\begin{array}{l}\text { If biological cycles are long, recent past } \\
\text { is a bad proxy for the future and } \\
\text { regulation can lead to repeated } \\
\text { collapses of fishery }\end{array}$ \\
\hline
\end{tabular}

economic rents, we simulate how well a regulator could estimate the periodicity using nonlinear least squares. Using a system with a random component, we start with a 10-year history of the system and then re-estimate the growth parameters in each year to predict the optimal policy stock level in the next period.

Note that fluctuations in the stock level are necessary to identify the system. If a government policy of a constant target stock was successful in fixing the fish stock at a certain level, it would be impossible to identify $\alpha_{0}$ and $\alpha_{1}$ separately as $F$ is a constant, and thus, the two would be completely collinear. ${ }^{18}$ Therefore, some variation in the observable stock is required. ${ }^{19}$ In a sense, the regulator can learn from mistakes. If parameters are estimated incorrectly, the harvest quota would be set incorrectly and result in deviations of the stock size away from the constant stock size $F^{*}$, which in turn helps to identify the system in future periods. While a constant $F$ does not allow a regulator to identify $\alpha_{0}, \alpha_{1}$, and the optimal stock level, residuals from a sufficiently long time series will allow the regulator to identify whether growth rates are time varying or not.

In case the fishery exhibits critical depensation, i.e., it requires a nonzero fish stock in order to survive, such "learning by doing" becomes problematic, as mistakes can become irreversible if the fish stock drops below the critical level where it can recover. It is preferable to use other strategies, e.g., harvesting a fraction of the stock to ensure that the fish stock does not fall below the minimum required stock level to sustain the species yet there is still variation in the observed fish stock to identify the system.

18 For illustrative purposes: $\alpha_{0}=0.2, \alpha_{1}=-0.1$ would give the same growth at the constant stock size $F=1$ as all other combinations of the form $\alpha_{0}=0.1-\alpha_{1}$ as long as $F=1$. One requires at least one observation of $F \neq 1$ to identify them separately.

19 Smith et al. (2007) present a structural model where data on catch and effort alone can help identify the latent stock variable under certain assumptions. 

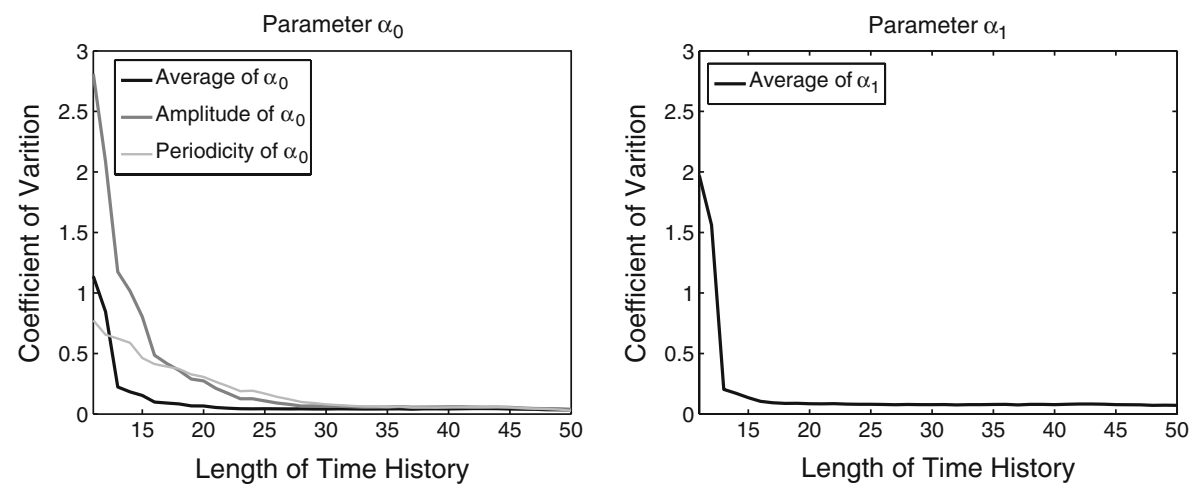

Fig. 7 Estimating the periodicity of the growth parameter. Notes: The above graphs display the coefficient of variation of the estimated model parameters under the periodically fluctuating growth equation. Note that longer histories reduce the uncertainty and result in lower standard deviations, though initially, there might be large swings, even though the simulated error component is rather small. The parameter values are $\omega=0.5, \theta=1, p=500, \delta=0.025, \alpha_{0}=0.5+0.1 \sin \left(\frac{2 \pi t}{50}\right), \alpha_{1}=-0.5, \sigma=0.01$

Figure 7 displays the coefficient of variation of repeatedly estimating the model parameters using non-linear least squares. ${ }^{20}$ Not surprisingly, these parameters are estimated more and more precisely as the history of a fish species increases. However, even a relatively small error component combined with a relatively small time history can lead to estimates that are far away from the true parameter.

There are three lessons for a fishing policy that incorporates cyclical population dynamics. First, the preferred data sets will have a time series of significant length. There may be little power to distinguish between different structural models if the time dimension of the data series is not long and/or the underlying source of the cyclical dynamics is not known. In these typical cases, it may be worth exploring time series models including those allowing for regime shifts in addition to, or in place of, the usual structural models (Hamilton 1989). ${ }^{21}$ Most fisheries do not have long time series, but there are some exceptions. For example, 130 years of data have been assembled for the Lofoten fishery in Norway (Hannesson et al. 2008).

Second, one indication of the pervasive nature of the dynamic forces in fisheries comes from looking at the data underlying the recent article by Costello et al. (2008). Their data includes total catches for approximately 7,000 fisheries with 10 or more years of data. Coding observations above the mean catch as 1 and those below the mean catch as -1 , one can run a regression of the recoded value $(1 /-1)$ in the current period on the recoded value in the previous period for each fishery. A model with i.i.d. error terms would suggest a coefficient of zero, as shocks in the current period are uncorrelated with shocks in the previous period.

\footnotetext{
20 We restrict both periodicity and offset of $\alpha_{0}$ to be integer-valued. The coefficient of variation is the standard deviation of the estimate divided by the true mean.

21 One difficulty with short time series is the well-known problem of separating out cycles and trends (Baxter and King 1999). In a fisheries model, the cycles may be driven by either the underlying physical/ecological forces or by the age-class structure of the fishery (Bjornstad et al. 1999). The trend component might be driven by technological change. Extreme events can alter both the cycle and trend.
} 
Yet, among all fisheries, the average coefficient is 0.61 . It is negative for less than $3 \%$ of the fisheries, suggesting that shocks are positively correlated in time. ${ }^{22}$

Third, the underlying physical/ecological processes influencing growth rates are not fully understood at this point. Our optimal policy is derived using a simplified model structure and should be seen as a motivating example why time-variant harvest rates might improve economic rents. Large scale data sets such as those assembled for the California Cooperative Oceanic Fisheries Investigations, which combine a range of fisheries data with ecological and physical indicators over the last 50 years, are facilitating work on how physical and ecological processes can influence fish populations (Rykaczewski and Checkley 2008).

\section{Stock-Dependent Harvest Cost—Multiple Species}

In the following we extend the analysis to include several species to highlight the importance of inter-species dynamics. ${ }^{23}$ Even when there are limited fluctuations in the biological growth function for a subset of the species, interspecies dynamics can lead to large fluctuations of the harvest rate for other species as well. Interspecies dynamics coupled with fluctuating biological growth parameters only amplifies the results of previous sections that the desired fish stock/harvest quotas should be time-varying. Moreover, looking at one species at a time can give a misleading picture.

The general setup of a three-species fish system with fish stocks $F_{1}(t), F_{2}(t)$, and $F_{3}(t)$ can be expressed using $\log \left(\dot{F}_{i}(t)\right) \equiv \frac{\dot{F}_{i}(t)}{F_{i}(t)}$

$$
\left[\begin{array}{l}
\log \left(\dot{F}_{1}(t)\right) \\
\log \left(\dot{F}_{2}(t)\right) \\
\log \left(\dot{F}_{3}(t)\right)
\end{array}\right]=\underbrace{\left[\begin{array}{l}
\alpha_{10} \\
\alpha_{20} \\
\alpha_{30}
\end{array}\right]}_{\mathbf{b}}+\underbrace{\left[\begin{array}{lll}
\alpha_{11} & \alpha_{12} & \alpha_{13} \\
\alpha_{21} & \alpha_{22} & \alpha_{23} \\
\alpha_{31} & \alpha_{23} & \alpha_{33}
\end{array}\right]}_{\mathbf{A}}\left[\begin{array}{l}
F_{1}(t) \\
F_{2}(t) \\
F_{3}(t)
\end{array}\right]
$$

In the following we assume that the matrix $\mathbf{A}$ has full rank and is invertible. ${ }^{24}$ The steadystate stocks without human interventions are given to be:

$$
\left[\widehat{F_{1}(t)}, \widehat{F_{2}(t)}, \widehat{F_{3}(t)}\right]^{\prime}=-\mathbf{A}^{-1} \mathbf{b}
$$

Let the two prey species be $F_{1}(t)$ and $F_{2}(t)$, and the predator species be $F_{3}(t)$. We use a revised Lotka-Volterra predator-prey system that allows for a reduction in the growth rate once the combined prey population gets too large and available food supplies diminish (Lotka 1925; Volterra 1931). We assume that $\alpha_{i 0}>0, \alpha_{i j}<0$, for $i \in\{0,1\} ; j \in\{1,2,3\}$, i.e., there is an intrinsic growth rate for prey fish which gets reduced by the number of fish in all species. On the other hand, $\alpha_{30}=0, \alpha_{31}>0, \alpha_{32}>0, \alpha_{33}<0$, i.e., the predator fish feeds exclusively on the two prey fish, the stock of which increase the growth rate of the

22 These results are subject to several caveats. First, one would ideally like to have an estimate of the stock size rather than catch. Second, one might worry that the correlation estimate is an artifact of trends in catch as a fishery is collapsing. However, a regression that allows for a linear time trend still finds an average coefficient on the previous period's shock of 0.48 and it is negative for only $6 \%$ of the fisheries. Third, the temporal correlation might also be due to auto-correlated shocks.

23 The implications of multi-species models with stationary growth parameters have received some attention in the economics literature, e.g., Hannesson (1983). Hollowed et al. (2000) gives a nice overview over multi-species fisheries in the biology literature.

24 In another paper we examine co-integrated fish species where the matrix $\mathbf{A}$ has less than rank three. If two species are co-integrated, there is no stable maximum sustainable harvest of one of the co-integrated species, as fishing one species alone will lead it to be replaced by the other co-integrated species. 
predator fish. There is again crowding out by the own species. Note that such a setup does not allow for "foraging," where animals strategically choose where to feed and live to obtain the highest survival probability. ${ }^{25}$

The multi-species system can be compared to the single-species model in the previous section. The fish stocks of other species act as shifters to the intrinsic growth rate, e.g., for fish species 1 , the analogous term to $\alpha_{0}$ in the single-species model now becomes $\alpha_{10}(t)+\alpha_{12} F_{2}(t)+\alpha_{13} F_{3}(t)$, while $\alpha_{1}$ is now labeled $\alpha_{11}$. Accordingly, the carrying capacity for species 1 is $K_{1}=\frac{\alpha_{10}(t)+\alpha_{12} F_{2}(t)+\alpha_{13} F_{3}(t)}{-\alpha_{11}}$, which cannot only fluctuate due to the possibly time-varying parameter $\alpha_{10}$, but also through varying population sizes of the other fish species. Even for the predator fish (species 3) which exclusively feeds on the prey fish (i.e., $\alpha_{30}=0$ ), the carrying capacity will become time-varying if the stock of the two prey fish fluctuate over time as $K_{3}=\frac{\alpha_{31} F_{1}(t)+\alpha_{32} F_{2}(t)}{-\alpha_{33}}$. By the same token, if the two food fish would not directly interact $\left(\alpha_{12}=\alpha_{21}=0\right)$, fluctuations in the biological growth parameter of one food fish would still impact the other food fish through common links with the predator fish (species 3). Once fish species are allowed to interact, fluctuations in the growth parameter of one species can translate directly or indirectly into fluctuations of other species.

Similar to previous sections, we abstract from uncertainty. Assume that fisheries management maximizes the present value of future harvests $h_{i}(t), i=1,2,3$. In the following we rely on the standard modelling framework where harvest $h_{i}(t)$ is a linear function of effort $e_{i}(t)$ and stock size $F_{i}(t)$, i.e., $h_{i}(t)=\theta_{i} e_{i}(t) F_{i}(t)$. Furthermore, there is a constant marginal cost of effort $\omega$ as well as constant fish price $p_{i}$. The regulator is maximizing the discounted value of future profits with discount rate $\delta$, i.e., the problem becomes

$$
\max _{h_{i}(t)} \int_{0}^{\infty} e^{-\delta t}\left[p_{1} h_{1}(t)-\omega \frac{h_{1}(t)}{\theta_{1} F_{1}(t)}+p_{2} h_{2}(t)-\omega \frac{h_{2}(t)}{\theta_{2} F_{2}(t)}+p_{3} h_{3}(t)-\omega \frac{h_{3}(t)}{\theta_{3} F_{3}(t)}\right] d t
$$

$$
\begin{array}{ll}
\text { s.t. } \dot{F}_{1}(t)=\left[\alpha_{10}+\alpha_{11} F_{1}(t)+\alpha_{12} F_{2}(t)+\alpha_{13} F_{3}(t)\right] F_{1}(t)-h_{1}(t) \\
\dot{F}_{2}(t)=\left[\alpha_{20}+\alpha_{21} F_{1}(t)+\alpha_{22} F_{2}(t)+\alpha_{23} F_{3}(t)\right] F_{2}(t)-h_{2}(t) \\
\dot{F}_{3}(t)=\left[\alpha_{30}+\alpha_{31} F_{1}(t)+\alpha_{32} F_{2}(t)+\alpha_{33} F_{3}(t)\right] F_{3}(t)-h_{3}(t)
\end{array}
$$

The derivation is similar to the single-species case in the previous section and given in the appendix.

Proposition 7 The desired stock levels in the multi-species system are given by

$$
\begin{aligned}
& -2 \alpha_{11} p_{1} \theta_{1} F_{1}^{2}+\theta_{1} p_{1}\left[\delta-\alpha_{10}\right]+\omega \alpha_{11}+\alpha_{21} \frac{\theta_{1}}{\theta_{2}}+\alpha_{31} \frac{\theta_{1}}{\theta_{3}} \\
& -\theta_{1}\left[\alpha_{12} p_{1}+\alpha_{21} p_{2}\right] F_{2}-\theta_{1}\left[\alpha_{13} p_{1}+\alpha_{31} p_{3}\right] F_{3} F_{1}-\delta \omega=0 \\
& -2 \alpha_{22} p_{2} \theta_{2} F_{2}^{2}+\theta_{2} p_{2}\left[\delta-\alpha_{20}\right]+\omega \alpha_{22}+\alpha_{12} \frac{\theta_{2}}{\theta_{1}}+\alpha_{32} \frac{\theta_{2}}{\theta_{3}} \\
& -\theta_{2}\left[\alpha_{21} p_{2}+\alpha_{12} p_{1}\right] F_{1}-\theta_{2}\left[\alpha_{23} p_{2}+\alpha_{32} p_{3}\right] F_{3} F_{2}-\delta \omega=0 \\
& -2 \alpha_{33} p_{3} \theta_{3} F_{3}^{2}+\theta_{3} p_{3}\left[\delta-\alpha_{30}\right]+\omega \alpha_{33}+\alpha_{23} \frac{\theta_{3}}{\theta_{2}}+\alpha_{13} \frac{\theta_{3}}{\theta_{1}} \\
& -\theta_{3}\left[\alpha_{32} p_{3}+\alpha_{23} p_{2}\right] F_{2}-\theta_{3}\left[\alpha_{31} p_{3}+\alpha_{13} p_{1}\right] F_{1} F_{3}-\delta \omega=0
\end{aligned}
$$

The idea is analogous to the single species case with logistic growth function $g(F)=$ $\left[\alpha_{0}(t)+\alpha_{1} F(t)\right] F(t)$ where $g^{\prime}\left(F^{*}\right)+\frac{\omega g\left(F^{*}\right)}{F^{*}\left(\theta p F^{*}-\omega\right)}=\delta$, except that the stock size $F_{j, j \neq i}$ of the fish populations different from species $i$ shifts the intrinsic growth rate $\alpha_{i 0}(t)$.

\footnotetext{
25 Numerical simulations methods for foraging arenas are outlined in Walters et al. (1997). They generally reduce short-term oscillations.
} 

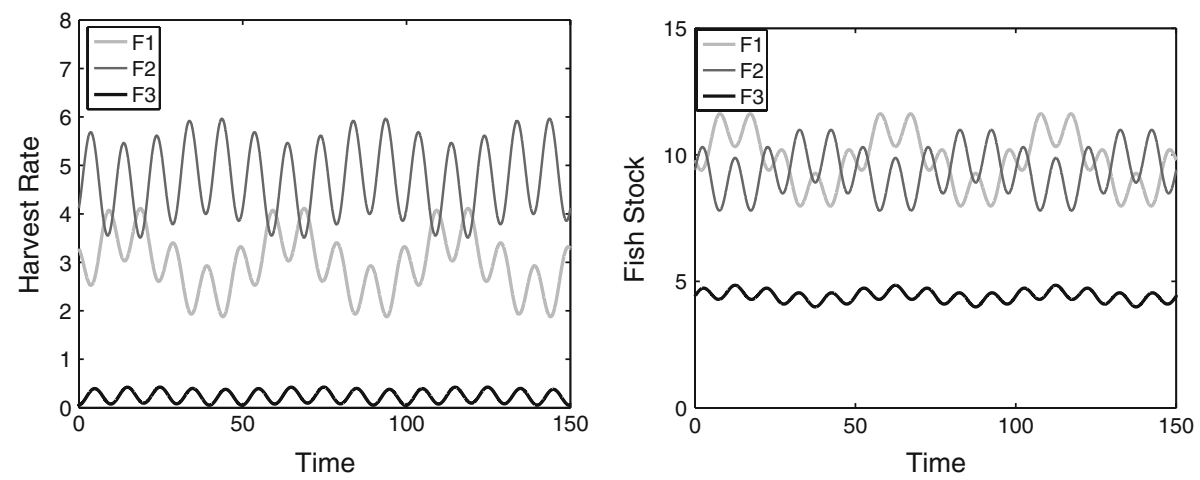

Fig. 8 Multi-species fishery system with oscillating growth rates. Notes: The left graph displays the optimal harvest rates while the right graph shows the resulting fish stocks. The intrinsic growth rates of the two prey fish oscillate according to $\alpha_{10}=0.8+0.04 \sin \left(\frac{2 \pi t}{50}\right), \alpha_{20}=1+0.05 \sin \left(\frac{2 \pi t}{10}\right)$. The economic parameters are $\theta_{1}=\theta_{2}=\theta_{3}=1, \omega=50$, and $p_{1}=p_{2}=100, p_{3}=500$. The discounted value of the harvest from the three species are $11,713,17,855$, and 4,687 , respectively

In the following we let the growth rates of the two prey fish oscillate by $5 \%$. The parameters are as follows: $\dot{F}_{1}=\left[0.8+0.04 \sin \left(\frac{2 \pi t}{50}\right)-0.025 F_{1}-0.025 F_{2}-0.004 F_{3}\right] F_{1}$ for food species 1 and $\dot{F}_{2}=\left[1+0.05 \sin \left(\frac{2 \pi t}{10}\right)-0.0125 F_{1}-0.0375 F_{2}-0.005 F_{3}\right] F_{2}$ for food species 2 as well as $\dot{F}_{3}=\left[0.003 F_{1}+0.004 F_{2}-0.003 F_{3}\right] F_{3}$ for the predator species. Furthermore, let $\theta_{1}=\theta_{2}=\theta_{3}=1, \omega=50$, and $p_{1}=p_{2}=100, p_{3}=500$, i.e., the predator fish is more valuable than the prey fish.

The optimal harvest quantities and resulting fish stocks are displayed in Fig. 8. There are several noteworthy features. First, the two prey species have growth rates that oscillate with different periodicities of 10 and 50 years, and each species oscillates with a combination of the two due to the interlinkage between species. Second, the unique stable equilibrium without human intervention under the average growth rates are [6.67, 20.00, 33.33], yet the optimal stock level of the predator species is significantly lower. The reason for this effect is that the predator species has a negative effect on the prey species, while the prey species on the other hand have a positive effect on the growth rate of the predator. In the economic equilibrium, the stock of the latter is reduced. This effect becomes less pronounced the larger the profit margin of the predator species. Third, even though growth rates of the prey species fluctuate by a relatively small $\pm 5 \%$, the optimal harvest rate of the predator species fluctuates between 0.049 and 0.43 , almost a nine-fold difference. This amplifies our main conclusions from the single-species model: even modest fluctuation can lead to strong time-varying harvest rates due to inter-species dynamics.

\section{Conclusions}

The classical fisheries model assumes time-invariant biological growth parameters with possibly i.i.d error terms, yet there is ample evidence that cyclical population dynamics are important at least for some commercial fisheries.

We show that cyclical population dynamics drive a further wedge between optimal economic policies and the biological concept of maximum sustainable yield underlying many regulations. Economists have long argued for limits on the allowable fishing quantity or 
constant target stocks to cope with the open-access problem, yet many regulations are still implicitly or explicitly based on the concept of MSY, i.e., the highest time-invariant harvest rate that can be sustained forever. In the standard model of the fishery, the harvest rate maximizing economic rents is identical to MSY for a zero discount rate and stockindependent harvest cost. While a non-zero discount rate and stock-dependent harvest cost drive the economist's recommendation away from the biologist's MSY, these factors work in opposite directions. ${ }^{26}$ However, once the growth rate is allowed to vary periodically, so do desired fish stocks. The optimal economic policy diverges from MSY, which by definition is time-invariant, even in the presence of a zero discount rate and stock-independent harvest cost.

Moreover, we show that not only is the optimal harvest rate cyclical, but that its cycle lags the one of the biological growth parameters. This has strong policy implications as the optimal policy is no longer directly related to the observed fish stock. Fishermen are quick to argue for higher quotas as soon as fish stocks are increasing, for the same reason that environmentalists advocate harvest reductions once fish stocks decline. Neither argument is accurate in the presence of cyclical population dynamics. Harvest closures occur during times when biological growth parameters are improving most rapidly as the return to not fishing is largest. This will occur after the biological growth function has bottomed out and already started to increase. Once we introduce inter-species dependence, modest fluctuations in the growth parameter in one species can lead to very large fluctuations in species that feed off it. Standard MSY fishing policies based on the average growth functions, or adaptive policies that periodically adjust the optimal harvest rate, can lead to repeated collapses in the fish stock as the inadequately large harvest limits will ruin the fish stock during consecutive years with low biological growth.

Our results contribute to understanding the economic implications of the ecosystem approach to management that has been recommended by the Pew Oceans Commission (2003) and many biologists. We consider the simple case where biological growth varies in a smooth and highly predictable way for both a single species and interlinked species. The results of a standard economic model of the fishery, which imbeds a time invariant growth rate, are surprisingly non-robust to this change.

There are two ways to expand upon our work. First, one could take our cyclical growth rate and make it a function of various environmental factors like temperature and salinity. This will introduce considerable complexity, particularly as one moves to the multi-species case, and the key issue is likely to be how predictable the growth rate is and at what time horizon. Still, the main result that the optimal harvest policy is time-variant and lags the cycle of the biological growth parameters should remain. Second, we have largely abstracted from the entry and exit decision into fisheries and capital adjustment cost that has received considerable attention from economists (e.g., Berck and Perloff 1984; Singh et al. 2006). How the dynamics of this process work in the face of a time varying growth rate is an open question and one with strong implications for the current generation of regulatory instruments proposed by economists including limited entry programs (Walker et al. 1990), individual transferable quotas (Newell et al. 2005) and landing fees (Weitzman 2002) because of the issues posed for the connection between fishing capacity and fish stocks (Kirkley et al. 2002).

Acknowledgements We thank anonymous referees, as well as Joshua Abbott, Christopher Costello, Geoffrey Heal, Jason Murray, Marty Smith, Dale Squires, Jim Wilen, and seminar participants at NOAA, Southwest

\footnotetext{
26 The economically optimal stock size is increasing in the former and decreasing in the latter. Because these two factors work in opposite directions, the stock level associated with MSY can still be a good approximation to the stock level which maximizes the economic rent associated with the resources.
} 
Fisheries Science Center, University of Nevada at Reno, UC Berkeley, UC Davis, and Yale University for helpful comments. Financial support from the Southwest Fisheries Science Center is gratefully acknowledged.

Open Access This article is distributed under the terms of the Creative Commons Attribution Noncommercial License which permits any noncommercial use, distribution, and reproduction in any medium, provided the original author(s) and source are credited.

\section{Appendix: Proofs}

Proof of Proposition 1 The optimal harvest policy $h(t)$ is to keep the fish stock at the desired level $F^{*}(t)=\frac{\alpha_{0}(t)-\delta(t)}{-2 \alpha_{1}(t)}$ if feasible, or set the harvest rate to zero until the desired fish stock is reached.

Proof The current-value Hamiltonian becomes

$$
\begin{aligned}
H & =[p-\omega] h(t)+\mu(t)\left\{\left[\alpha_{0}(t)+\alpha_{1}(t) F(t)\right] F(t)-h(t)\right\} \\
& =[p-\omega-\mu(t)] h(t)+\mu(t)\left[\alpha_{0}(t)+\alpha_{1}(t) F(t)\right] F(t)
\end{aligned}
$$

Note that the Hamiltonian is a linear function in the harvest rate $h(t)$. If the multiplicative term on the harvest rate is different from zero, one would either set the harvest rate to zero or infinite. The necessary conditions for $h(t)$ imply that

$$
h(t)= \begin{cases}0, & \text { if } p-\omega-\mu(t)<0 \\ \infty, & \text { if } p-\omega-\mu(t)>0 \\ \text { see below, } & \text { if } p-\omega-\mu(t)=0\end{cases}
$$

In the following consider first the case where $p-\omega-\mu(t)=0$, which implies that $\dot{\mu}(t)=0$, or $\mu(t)=\bar{\mu}$. Differentiating the Hamiltonian with respect to the state variable $F(t)$ gives

$$
H_{F}=\mu(t)\left[\alpha_{0}(t)+2 \alpha_{1}(t) F(t)\right]=\delta(t) \mu(t)-\underbrace{\dot{\mu}(t)}_{0}
$$

Since $\bar{\mu}>0$ (a larger fish stock can always be fished down instantaneously for a profit) we know that $\alpha_{0}(t)+2 \alpha_{1}(t) F(t)-\delta(t)=0$, and hence, $F^{*}(t)=\frac{\alpha_{0}(t)-\delta(t)}{-2 \alpha_{1}(t)}$.

Now consider the two extreme cases: decreasing the fish stock increases the shadow value due to the assumed concave logistic growth function. Letting $\mu(t)$ fall below $\bar{\mu}$ can never be optimal in this deterministic model as one should increase fishing efforts. The converse is not true: While one can instantly draw down the fish stock as much as desired, one cannot increase it an arbitrary speed as harvest is non-negative and fish growth is limited. So if $\mu(t)$ rises above $\bar{\mu}$, it becomes optimal to set the harvest rate to zero.

Extension: If the regulator maximizes the log of profits, the predicted fluctuations in the desired fish stock will be smoothed out.

The current-value Hamiltonian becomes

$$
H=\ln [p-\omega]+\ln [h(t)]+\mu(t)\left\{\left[\alpha_{0}(t)+\alpha_{1}(t) F(t)\right] F(t)-h(t)\right\}
$$

The first order conditions are:

$$
\begin{aligned}
& \frac{\partial H}{\partial h}=\frac{1}{h}-\mu=0 \Rightarrow \mu=\frac{1}{h} \Rightarrow \dot{\mu}=-\frac{\dot{h}}{h^{2}} \\
& \frac{\partial H}{\partial F}=\mu\left[\alpha_{0}+2 \alpha_{1} F\right]=\delta \mu-\dot{\mu}
\end{aligned}
$$


Using the expression for $\mu$ and $\dot{\mu}$ from $\frac{\partial H}{\partial h}$ in $\frac{\partial H}{\partial F}$ gives

$$
\frac{1}{h}\left[\alpha_{0}+2 \alpha_{1} F\right]=\delta \frac{1}{h}+\frac{\dot{h}}{h^{2}} \Leftrightarrow F=\frac{\alpha_{0}(t)-\delta(t)-\frac{\dot{h}}{h}}{-2 \alpha_{1}(t)}
$$

The difference to proceeding Proposition 1 is the additional term $\frac{\dot{h}}{h}$ in the numerator. Previously, when the desired fish stock $F_{\text {discountedprofit }}^{*}$ increases, eventually so does the harvest rate $\dot{h}>0$ and there is an incentive to let $F$ (and hence $h$ ) grow at a slower rate. An analogous argument holds for the case where the desired fish stock is decreasing.

Proof of Proposition 2 The minimum amplitude $\alpha_{02}$ for which there is a harvest closure in the cyclic single-species model is increasing in the intrinsic growth rate $\alpha_{01}$ and periodicity $\alpha_{04}$, and decreasing in the interest rate $\delta$.

Proof The change in the desired stock level is $\dot{F}^{*}(t)=\frac{\alpha_{0}^{\prime}(t)}{-2 \alpha_{1}}$. The growth rate at the desired stock $F^{*}$ is $g\left(F^{*}(t)\right)=\left[\alpha_{0}(t)+\alpha_{1} F^{*}(t)\right] F^{*}(t)=\frac{\alpha_{0}(t)^{2}-\delta^{2}}{-4 \alpha_{1}}$. If the former exceeds the latter, i.e., if the required increase in the stock level cannot be sustained by natural growth, the harvest rate will become zero, i.e.,

$$
\alpha_{0}(t)^{2}-\delta^{2} \leq 2 \alpha_{0}^{\prime}(t) \Leftrightarrow \alpha_{0}(t)^{2}-\delta^{2}-2 \alpha_{0}^{\prime}(t) \leq 0
$$

The first order condition for minimizing $\alpha_{0}(t)^{2}-\delta^{2}-2 \alpha_{0}^{\prime}(t)$ gives $2 \alpha_{0}(t) \alpha_{0}^{\prime}(t)-2 \alpha_{0}^{\prime \prime}(t)=0$. Using $\alpha_{0}(t)=\alpha_{01}+\alpha_{02} \sin \left(\frac{2 \pi\left(t+\alpha_{03}\right)}{\alpha_{04}}\right)$ we have for the solution $\alpha_{0}(t)=\frac{\alpha_{0}^{\prime \prime}(t)}{\alpha_{0}^{\prime}(t)}=-\frac{2 \pi}{\alpha_{04}}$ $\tan (x)$, where $x=\frac{2 \pi\left[t-\alpha_{03}\right]}{\alpha_{04}}$. Since $\alpha_{0}(t)>0$ the shape of $-\tan (x)$ implies that there are two possible solutions, $x_{1}^{*} \in\left(-\frac{\pi}{2}, 0\right)$ and $x_{2}^{*} \in\left(\frac{\pi}{2}, \pi\right)$.

Using $\sin \left(x_{1}^{*}\right)<0, \cos \left(x_{1}^{*}\right)>0$ and $\sin \left(x_{2}^{*}\right)>0, \cos \left(x_{2}^{*}\right)<0$ we know that $x_{1}^{*}$ is a minimum and $x_{2}^{*}$ is a maximum. In the remainder we limit the analysis to $x^{*}=x_{1}^{*} \in\left(-\frac{\pi}{2}, 0\right)$. Totally differentiating $\alpha_{01}+\alpha_{02} \sin \left(x^{*}\right)+\frac{2 \pi}{\alpha_{04}} \tan \left(x^{*}\right)=0$ we get

$$
\begin{gathered}
\{1\} d \alpha_{01}+\left\{\sin \left(x^{*}\right)\right\} d \alpha_{02}-\left\{\frac{2 \pi}{\alpha_{04}^{2}} \tan \left(x^{*}\right)\right\} d \alpha_{04} \\
+\left\{\alpha_{02} \cos \left(x^{*}\right)+\frac{2 \pi}{\alpha_{04} \cos \left(x^{*}\right)^{2}}\right\} d x^{*}=0
\end{gathered}
$$

The derivatives of $x^{*}$ with respect to the parameters of the synodal growth function are, recalling that $x^{*} \in\left(\frac{\pi}{2}, 0\right)$ :

$$
\begin{aligned}
& \frac{d x^{*}}{d \alpha_{01}}=\frac{-1}{\alpha_{02} \cos \left(x^{*}\right)+\frac{2 \pi}{\alpha_{04} \cos \left(x^{*}\right)^{2}}}<0 \\
& \frac{d x^{*}}{d \alpha_{02}}=\frac{-\sin \left(x^{*}\right)}{\alpha_{02} \cos \left(x^{*}\right)+\frac{2 \pi}{\alpha_{04} \cos \left(x^{*}\right)^{2}}}>0 \\
& \frac{d x^{*}}{d \alpha_{04}}=\frac{\frac{2 \pi}{\alpha_{04}^{2}} \tan \left(x^{*}\right)}{\alpha_{02} \cos \left(x^{*}\right)+\frac{2 \pi}{\alpha_{04} \cos \left(x^{*}\right)^{2}}}<0
\end{aligned}
$$

Call the minimum amplitude were the harvest rate becomes zero as $\overline{\alpha_{02}}$. Thus, we have

$$
M=\left[\alpha_{01}+\overline{\alpha_{02}} \sin \left(x^{*}\right)\right]^{2}-\delta^{2}-\frac{4 \pi \overline{\alpha_{02}}}{\alpha_{04}} \cos \left(x^{*}\right)
$$


We have

$$
\begin{aligned}
\frac{d M}{d \overline{\alpha_{02}}}= & 2\left[\alpha_{01}+\overline{\alpha_{02}} \sin \left(x^{*}\right)\right]\left[\sin \left(x^{*}\right)+\overline{\alpha_{02}} \cos \left(x^{*}\right) \frac{d x^{*}}{d \overline{\alpha_{02}}}\right] \\
& -\frac{4 \pi}{\alpha_{04}} \cos \left(x^{*}\right)+\frac{4 \pi \overline{\alpha_{02}}}{\alpha_{04}} \sin \left(x^{*}\right) \frac{d x^{*}}{d \overline{\alpha_{02}}} \\
= & \frac{4 \pi \sin \left(x^{*}\right)\left[\alpha_{01}+\overline{\alpha_{02}} \sin \left(x^{*}\right)\right]}{\overline{\alpha_{02}} \alpha_{04} \cos \left(x^{*}\right)^{3}+2 \pi} \\
& -\frac{4 \pi}{\alpha_{04}} \cos \left(x^{*}\right)-\frac{4 \pi \overline{\alpha_{02}} \sin \left(x^{*}\right)^{2}}{\alpha_{04}\left[\overline{\alpha_{02}} \cos \left(x^{*}\right)+\frac{2 \pi}{\alpha_{04} \cos \left(x^{*}\right)^{2}}\right]}<0 \\
\frac{d M}{d \alpha_{01}}= & 2\left[\alpha_{01}+\overline{\alpha_{02}} \sin \left(x^{*}\right)\right]\left[1+\overline{\alpha_{02}} \cos \left(x^{*}\right) \frac{d x^{*}}{d \alpha_{01}}\right]+\frac{4 \pi \overline{\alpha_{02}}}{\alpha_{04}} \sin \left(x^{*}\right) \frac{d x^{*}}{d \alpha_{01}} \\
= & \frac{4 \pi\left[\alpha_{01}+\overline{\alpha_{02}} \sin \left(x^{*}\right)\right]}{\overline{\alpha_{02}} \alpha_{04} \cos \left(x^{*}\right)^{3}+2 \pi}-\frac{\sin \left(x^{*}\right)}{\alpha_{04}\left[\overline{\alpha_{02}} \cos \left(x^{*}\right)+\frac{2 \pi}{\alpha_{04} \cos \left(x^{*}\right)^{2}}\right]}>0
\end{aligned}
$$

Finally, using $\alpha_{01}+\alpha_{02} \sin \left(x^{*}\right)+\frac{2 \pi}{\alpha_{04}} \tan \left(x^{*}\right)=0$ we get

$$
\begin{aligned}
\frac{d M}{d \alpha_{04}} & =2\left[\alpha_{01}+\overline{\alpha_{02}} \sin \left(x^{*}\right)\right] \overline{\alpha_{02}} \cos \left(x^{*}\right) \frac{d x^{*}}{d \alpha_{04}}+\frac{4 \pi \overline{\alpha_{02}}}{\alpha_{04}^{2}} \cos \left(x^{*}\right)+\frac{4 \pi \overline{\alpha_{02}}}{\alpha_{04}} \sin \left(x^{*}\right) \frac{d x^{*}}{d \alpha_{04}} \\
& =-\frac{4 \pi \overline{\alpha_{02}}}{\alpha_{04}} \tan \left(x^{*}\right) \cos \left(x^{*}\right) \frac{d x^{*}}{d \alpha_{04}}+\frac{4 \pi \overline{\alpha_{02}}}{\alpha_{04}^{2}} \cos \left(x^{*}\right)+\frac{4 \pi \overline{\alpha_{02}}}{\alpha_{04}} \sin \left(x^{*}\right) \frac{d x^{*}}{d \alpha_{04}} \\
& =\frac{4 \pi \overline{\alpha_{02}}}{\alpha_{04}^{2}} \cos \left(x^{*}\right)>0 \\
\frac{d M}{d \delta} & =-2 \delta<0
\end{aligned}
$$

where $M$ is increasing in both $\alpha_{01}$ and $\alpha_{04}$, yet decreasing in $\overline{\alpha_{02}}$ and $\delta$.

Proof of Proposition 3 The desired fish stock $F^{*}(t)$ fluctuates synchronously with the biological growth parameter $\alpha_{0}(t)$, but the cycle of the optimal harvest rate lags the cycle of the biological growth parameter.

Proof First, the desired fish stock is $F^{*}(t)=\frac{\alpha_{0}(t)-\delta(t)}{-2 \alpha_{1}}$ by Proposition 1 thus the desired fish stock varies synchronously with $\alpha_{0}(t)$.

Second, the harvest rate is given by the difference between the growth rate and the change in desired stock level, i.e.,

$$
h(t)=\max \left\{\frac{\alpha_{0}(t)^{2}-\delta^{2}-2 \alpha_{0}^{\prime}(t)}{-4 \alpha_{1}}, 0\right\}
$$

In case the harvest rate is nonzero, we have

$$
h^{\prime}(t)=\frac{2 \alpha_{0}(t) \alpha_{0}^{\prime}(t)-2 \alpha_{0}^{\prime \prime}(t)}{-4 \alpha_{1}}
$$

When the biological growth function is at its maximum we have $\alpha_{0}^{\prime}(t)=0$ and $\alpha_{0}^{\prime \prime}(t)<0$ and hence the harvest rate is still increasing. When the biological growth function is at its minimum we have $\alpha_{0}^{\prime}(t)=0$ and $\alpha_{0}^{\prime \prime}(t)>0$ and the harvest rate is still decreasing. 
In case the harvest rate becomes zero, Proposition 2 has shown that the harvest rate becomes zero on $\left(\alpha_{03}-\frac{\pi}{2}, \alpha_{03}\right)$, while the biological growth function is at its lowest at $\alpha_{03}-\frac{\pi}{2}$.

Proof of Proposition 4 The optimal harvest policy $h(t)$ is to keep the fish stock at the desired level $F^{*}(t)=\frac{\theta p\left[\alpha_{0}(t)-\delta\right]-\alpha_{1} \omega}{-4 \alpha_{1} \theta p}+\sqrt{\left(\frac{\theta p\left[\alpha_{0}(t)-\delta\right]-\alpha_{1} \omega}{-4 \alpha_{1} \theta p}\right)^{2}+\frac{\delta \omega}{-2 \alpha_{1} \theta p}}$ if feasible, or set the harvest rate to zero until the desired fish stock is reached.

Proof The current-value Hamiltonian becomes

$$
\begin{aligned}
H & =p h(t)-\omega \frac{h(t)}{\theta F(t)}+\mu(t)\left\{\left[\alpha_{0}(t)+\alpha_{1} F(t)\right] F(t)-h(t)\right\} \\
& =\left[p-\frac{\omega}{\theta F(t)}-\mu(t)\right] h(t)+\mu(t)\left[\alpha_{0}(t)+\alpha_{1} F(t)\right] F(t)
\end{aligned}
$$

The necessary conditions for $h(t)$ imply that

$$
h(t)= \begin{cases}0, & \text { if } p-\frac{\omega}{\theta F(t)}-\mu(t)<0 \\ h_{\max }, & \text { if } p-\frac{\omega}{\theta F(t)}-\mu(t)>0 \\ \text { see below, } & \text { if } p-\frac{\omega}{\theta F(t)}-\mu(t)=0\end{cases}
$$

There are two border conditions. In the following consider the case where $p-\frac{\omega}{\theta F(t)}-\mu=0$, which implies that

$$
\dot{\mu}(t)=\frac{\omega}{\theta F(t)^{2}} \dot{F}(t)
$$

Differentiating the Hamiltonian with respect to the state variable $F(t)$ gives

$$
H_{F}=\frac{\omega h(t)}{\theta F(t)^{2}}+\mu(t)\left[\alpha_{0}(t)+2 \alpha_{1} F(t)\right]=\delta \mu(t)-\dot{\mu}(t)
$$

Using the expression for $\mu(t)$ and $\dot{\mu}(t)$ we get

$$
\begin{aligned}
& \frac{\omega h(t)}{\theta F(t)^{2}}+\dot{\mu}(t)=\mu(t)\left[\delta-\alpha_{0}(t)-2 \alpha_{1} F(t)\right] \\
\Leftrightarrow & \omega \frac{\dot{F}(t)+h(t)}{\theta F(t)^{2}}=\frac{\theta p F(t)-\omega}{\theta F(t)}\left[\delta-\alpha_{0}(t)-2 \alpha_{1} F(t)\right] \\
\Leftrightarrow & \omega \frac{\left[\alpha_{0}(t)+\alpha_{1} F(t)\right] F(t)-h(t)+h(t)}{F(t)}=[\theta p F(t)-\omega]\left[\delta-\alpha_{0}(t)-2 \alpha_{1} F(t)\right] \\
\Leftrightarrow & \omega \alpha_{0}(t)+\omega \alpha_{1} F(t)=\theta p F(t)\left[\delta-\alpha_{0}(t)\right]-2 \alpha_{1} \theta p F(t)^{2}-\omega \delta+\omega \alpha_{0}(t)+2 \alpha_{1} \omega F(t) \\
\Leftrightarrow & -2 \alpha_{1} \theta p F(t)^{2}+\left[\theta p\left[\delta-\alpha_{0}(t)\right]+\alpha_{1} \omega\right] F(t)-\omega \delta=0
\end{aligned}
$$

The only positive solution to this quadratic formula is (Note that $\alpha_{1}<0$ )

$$
F^{*}(t)=\underbrace{\frac{\theta p\left[\alpha_{0}(t)-\delta\right]-\alpha_{1} \omega}{-4 \alpha_{1} \theta p}}_{>0}+\sqrt{\underbrace{\left(\frac{\theta p\left[\alpha_{0}(t)-\delta\right]-\alpha_{1} \omega}{-4 \alpha_{1} \theta p}\right)^{2}}_{>0}+\underbrace{\frac{\delta \omega}{-2 \alpha_{1} \theta p}}_{>0}}
$$


Proof of Proposition 5 The desired stock level is increasing in the growth parameters $\alpha_{0}$ and cost of effort $\omega$, while it is decreasing in the growth parameter $-\alpha_{1}$, effort factor $\theta$ and price of fish $p$.

Proof We assume that the instantaneous growth rate $\alpha_{0}(t)>\delta \forall t$, and hence, $\alpha_{0}(t)-\delta>0$. The optimal stock level is given by:

$$
\begin{aligned}
F^{*}(t) & =\frac{\theta p\left[\alpha_{0}(t)-\delta\right]-\alpha_{1} \omega}{-4 \alpha_{1} \theta p}+\sqrt{\left(\frac{\theta p\left[\alpha_{0}(t)-\delta\right]-\alpha_{1} \omega}{-4 \alpha_{1} \theta p}\right)^{2}+\frac{\delta \omega}{-2 \alpha_{1} \theta p}} \\
& =\frac{\alpha_{0}-\delta}{-4 \alpha_{1}}+\frac{\omega}{4 \theta p}+\sqrt{\left(\frac{\alpha_{0}-\delta}{-4 \alpha_{1}}+\frac{\omega}{4 \theta p}\right)^{2}+\frac{\delta \omega}{-2 \alpha_{1} \theta p}}
\end{aligned}
$$

We have (recall that $\alpha_{1}<0$ )

$$
\begin{aligned}
& \frac{\partial F^{*}(t)}{\partial \alpha_{0}}=\frac{1}{-4 \alpha_{1}}\left[1+\frac{\frac{\alpha_{0}-\delta}{-4 \alpha_{1}}+\frac{\omega}{4 \theta p}}{\sqrt{\left(\frac{\alpha_{0}-\delta}{-4 \alpha_{1}}+\frac{\omega}{4 \theta p}\right)^{2}+\frac{\delta \omega}{-2 \alpha_{1} \theta p}}}\right]>0 \\
& \frac{\partial F^{*}(t)}{\partial \alpha_{1}}=\frac{\alpha_{0}-\delta}{4 \alpha_{1}^{2}}\left[1+\frac{\frac{\alpha_{0}-\delta}{-4 \alpha_{1}}+\frac{\omega}{4 \theta p}+\frac{\delta \omega}{\left[\alpha_{0}-\delta\right] \theta p}}{\sqrt{\left(\frac{\alpha_{0}-\delta}{-4 \alpha_{1}}+\frac{\omega}{4 \theta p}\right)^{2}+\frac{\delta \omega}{-2 \alpha_{1} \theta p}}}\right]>0 \\
& \frac{\partial F^{*}(t)}{\partial \omega}=\frac{1}{4 \theta p}\left[1+\frac{\frac{\alpha_{0}-\delta}{-4 \alpha_{1}}+\frac{\omega}{4 \theta p}+\frac{\delta}{-\alpha_{1}}}{\left.\sqrt{\left(\frac{\alpha_{0}-\delta}{-4 \alpha_{1}}+\frac{\omega}{4 \theta p}\right)^{2}+\frac{\delta \omega}{-2 \alpha_{1} \theta p}}\right]>0}\right. \\
& \frac{\partial F^{*}(t)}{\partial \theta}=-\frac{\omega}{4 \theta^{2} p}\left[1+\frac{\frac{\alpha_{0}-\delta}{-4 \alpha_{1}}+\frac{\omega}{4 \theta p}+\frac{\delta}{-\alpha_{1}}}{\sqrt{\left(\frac{\alpha_{0}-\delta}{-4 \alpha_{1}}+\frac{\omega}{4 \theta p}\right)^{2}+\frac{\delta \omega}{-2 \alpha_{1} \theta p}}}\right]<0 \\
& \frac{\partial F^{*}(t)}{\partial p}=-\frac{\omega}{4 \theta p^{2}}\left[1+\frac{\frac{\alpha_{0}-\delta}{-4 \alpha_{1}}+\frac{\omega}{4 \theta p}-\frac{\delta}{\alpha_{1}}}{\sqrt{\left(\frac{\alpha_{0}-\delta}{-4 \alpha_{1}}+\frac{\omega}{4 \theta p}\right)^{2}+\frac{\delta \omega}{-2 \alpha_{1} \theta p}}}\right]<0
\end{aligned}
$$

Proof of Proposition 6 The desired fish stock $F^{*}(t)$ varies synchronously with the periodic biological growth function, while the cycle of the harvest policy $h(t)$ lags behind the cycle of the biological growth function.

The desired stock level is

$$
F^{*}(t)=\frac{\theta p\left[\delta-\alpha_{0}(t)\right]+\alpha_{1} \omega}{4 \alpha_{1} \theta p}+\sqrt{\left(\frac{\theta p\left[\delta-\alpha_{0}(t)\right]+\alpha_{1} \omega}{4 \alpha_{1} \theta p}\right)^{2}-\frac{\delta \omega}{2 \alpha_{1} \theta p}}
$$


which varies with $\alpha_{0}(t)$ and the optimal stock level fluctuates synchronously with the biological growth function. Furthermore, we have

$$
\begin{aligned}
\frac{d F^{*}(t)}{d t} & =\frac{-\alpha_{0}^{\prime}(t)}{4 \alpha_{1}}\left[1+\frac{\frac{\theta p\left[\delta-\alpha_{0}(t)\right]+\alpha_{1} \omega}{4 \alpha_{1} \theta p}}{\sqrt{\left(\frac{\theta p\left[\delta-\alpha_{0}(t)\right]+\alpha_{1} \omega}{4 \alpha_{1} \theta p}\right)^{2}-\frac{\delta \omega}{2 \alpha_{1} \theta p}}}\right] \\
& =\frac{-\alpha_{0}^{\prime}(t)}{4 \alpha_{1}}\left[1+\frac{1}{\sqrt{1-\frac{8 \alpha_{1} \theta p \delta \omega}{\left[\theta p\left[\delta-\alpha_{0}(t)\right]+\alpha_{1} \omega\right]^{2}}}}\right] \\
\frac{d^{2} F^{*}(t)}{d t^{2}} & =\frac{-\alpha_{0}^{\prime \prime}(t)}{4 \alpha_{1}}\left[1+\frac{1}{\sqrt{1-\frac{8 \alpha_{1} \theta p \delta \omega}{\left[\theta p\left[\delta-\alpha_{0}(t)\right]+\alpha_{1} \omega\right]^{2}}}}\right]-\alpha_{0}^{\prime}(t)^{2} \frac{\frac{2 \theta^{2} p^{2} \delta \omega}{\left[\theta p\left[\delta-\alpha_{0}(t)\right]+\alpha_{1} \omega\right]^{3}}}{\left[1-\frac{8 \alpha_{1} \theta p \delta \omega}{\left[\theta p\left[\delta-\alpha_{0}(t)\right]+\alpha_{1} \omega\right]^{2}}\right]^{\frac{3}{2}}}
\end{aligned}
$$

The desired stock level $F^{*}(t)$ peaks at the same time as the cyclical biological growth function as $\frac{d F^{*}(t)}{d t}=0$ if and only if $\alpha_{0}^{\prime}(t)=0$.

The optimal non-zero harvest rate (when the system is not in a corner solution due to the non-negativity of the harvest rate) is

$$
h(t)=g\left(F^{*}(t)\right)-\frac{d F^{*}(t)}{d t}=\left[\alpha_{0}(t)+\alpha_{1} F^{*}(t)\right] F^{*}(t)-\frac{d F^{*}(t)}{d t}
$$

and therefore,

$$
h^{\prime}(t)=\alpha_{0}^{\prime}(t) F^{*}(t)+\left[\alpha_{0}(t)+2 \alpha_{1} F^{*}(t)\right] \frac{d F^{*}(t)}{d t}-\frac{d^{2} F^{*}(t)}{d t^{2}}
$$

If the biological growth function peaks we have $\alpha^{\prime}(t)=\frac{d F^{*}(t)}{d t}=0$ and $h^{\prime}(t)=-\frac{d^{2} F^{*}(t)}{d t^{2}}>$ 0 , i.e., the optimal harvest rate is still increasing.

On the other hand, if the biological growth rate is at its minimum, we again have $\alpha^{\prime}(t)=$ $\frac{d F^{*}(t)}{d t}=0$, but the sinus function is now in the convex portion and $h^{\prime}(t)=-\frac{d^{2} F^{*}(t)}{d t^{2}}<0$, i.e., the optimal harvest rate is still decreasing (assuming it is not a corner solution at zero to begin with).

Proof of Proposition 7 The desired stock levels in the multi-species system are given by

$$
\begin{aligned}
& -2 \alpha_{11} p_{1} \theta_{1} F_{1}^{2}+\theta_{1} p_{1}\left[\delta-\alpha_{10}\right]+\omega \alpha_{11}+\alpha_{21} \frac{\theta_{1}}{\theta_{2}}+\alpha_{31} \frac{\theta_{1}}{\theta_{3}} \\
& -\theta_{1}\left[\alpha_{12} p_{1}+\alpha_{21} p_{2}\right] F_{2}-\theta_{1}\left[\alpha_{13} p_{1}+\alpha_{31} p_{3}\right] F_{3} F_{1}-\delta \omega=0 \\
& -2 \alpha_{22} p_{2} \theta_{2} F_{2}^{2}+\theta_{2} p_{2}\left[\delta-\alpha_{20}\right]+\omega \alpha_{22}+\alpha_{12} \frac{\theta_{2}}{\theta_{1}}+\alpha_{32} \frac{\theta_{2}}{\theta_{3}} \\
& -\theta_{2}\left[\alpha_{21} p_{2}+\alpha_{12} p_{1}\right] F_{1}-\theta_{2}\left[\alpha_{23} p_{2}+\alpha_{32} p_{3}\right] F_{3} F_{2}-\delta \omega=0 \\
& -2 \alpha_{33} p_{3} \theta_{3} F_{3}^{2}+\theta_{3} p_{3}\left[\delta-\alpha_{30}\right]+\omega \alpha_{33}+\alpha_{23} \frac{\theta_{3}}{\theta_{2}}+\alpha_{13} \frac{\theta_{3}}{\theta_{1}} \\
& -\theta_{3}\left[\alpha_{32} p_{3}+\alpha_{23} p_{2}\right] F_{2}-\theta_{3}\left[\alpha_{31} p_{3}+\alpha_{13} p_{1}\right] F_{1} F_{3}-\delta \omega=0
\end{aligned}
$$


The Hamiltonian for the multi-species model is

$$
\begin{aligned}
H & =\sum_{i=1}^{3} p_{i} h_{i}(t)-\omega \frac{h_{i}(t)}{\theta_{i} F_{i}(t)}+\mu_{i}(t)\left\{\left[\alpha_{i 0}+\alpha_{i 1} F_{1}(t)+\alpha_{i 2} F_{2}(t)+\alpha_{i 3} F_{3}(t)\right] F_{i}(t)-h_{i}(t)\right\} \\
& =\sum_{i=1}^{3}\left[p_{i}-\frac{\omega}{\theta_{i} F_{i}(t)}-\mu_{i}(t)\right] h_{i}(t)+\mu_{i}(t)\left[\alpha_{i 0}+\alpha_{i 1} F_{1}(t)+\alpha_{i 2} F_{2}(t)+\alpha_{i 3} F_{3}(t)\right] F_{i}(t)
\end{aligned}
$$

The necessary conditions for $h_{i}(t)$ implies that

$$
h_{i}(t)= \begin{cases}0, & \text { if } p_{i}-\frac{\omega}{\theta_{i} F_{i}(t)}-\mu_{i}(t)<0 \\ h_{i, \max }, & \text { if } p_{i}-\frac{\omega}{\theta_{i} F_{i}(t)}-\mu_{i}(t)>0 \\ \text { see below, } & \text { if } p_{i}-\frac{\omega}{\theta_{i} F_{i}(t)}-\mu_{i}(t)=0\end{cases}
$$

In the following consider the case where $p_{i}-\frac{\omega}{\theta_{i} F_{i}(t)}-\mu_{i}(t)=0$, which implies that $\dot{\mu}_{i}(t)=\frac{\omega}{\theta_{i} F_{i}(t)^{2}} \dot{F}_{i}(t)$

Differentiating the Hamiltonian with respect to the state variables $F_{i}(t)$ gives

$$
\begin{aligned}
H_{F_{1}}= & \frac{\omega h_{1}(t)}{\theta_{1} F_{1}(t)^{2}}+\mu_{1}(t)\left[\alpha_{10}+2 \alpha_{11} F_{1}(t)+\alpha_{12} F_{2}(t)+\alpha_{13} F_{3}(t)\right] \\
& +\alpha_{21} \mu_{2}(t) F_{2}(t)+\alpha_{31} \mu_{3}(t) F_{3}(t)=\delta \mu_{1}(t)-\dot{\mu}_{1}(t) \\
H_{F_{2}}= & \frac{\omega h_{2}(t)}{\theta_{2} F_{2}(t)^{2}}+\mu_{2}(t)\left[\alpha_{20}+\alpha_{21} F_{1}(t)+2 \alpha_{22} F_{2}(t)+\alpha_{23} F_{3}(t)\right] \\
& +\alpha_{12} \mu_{1}(t) F_{1}(t)+\alpha_{32} \mu_{3}(t) F_{3}(t)=\delta \mu_{2}(t)-\dot{\mu}_{2}(t) \\
H_{F_{3}}= & \frac{\omega h_{3}(t)}{\theta_{3} F_{3}(t)^{2}}+\mu_{3}(t)\left[\alpha_{30}+\alpha_{31} F_{1}(t)+\alpha_{32} F_{2}(t)+2 \alpha_{33} F_{3}(t)\right] \\
& +\alpha_{13} \mu_{1}(t) F_{1}(t)+\alpha_{23} \mu_{2}(t) F_{2}(t)=\delta \mu_{3}(t)-\dot{\mu}_{3}(t)
\end{aligned}
$$

Substituting the expression for $\mu_{i}(t)$ and $\dot{\mu}_{i}(t)$ in the first equation

$$
\begin{aligned}
\delta \frac{p_{1} \theta_{1} F_{1}-\omega}{\theta_{1} F_{1}}-\frac{\omega}{\theta_{1} F_{1}^{2}} \dot{F}_{1}= & \frac{\omega h_{1}}{\theta_{1} F_{1}^{2}}+\frac{p_{1} \theta_{1} F_{1}-\omega}{\theta_{1} F_{1}}\left[\alpha_{10}+2 \alpha_{11} F_{1}+\alpha_{12} F_{2}+\alpha_{13} F_{3}\right] \\
& +\alpha_{21} \frac{p_{2} \theta_{2} F_{2}-\omega}{\theta_{2} F_{2}} F_{2}+\alpha_{31} \frac{p_{3} \theta_{3} F_{3}-\omega}{\theta_{3} F_{3}} F_{3}
\end{aligned}
$$

Which yields after multiplying through by $\theta_{1} F_{1}$

$$
\begin{aligned}
\delta\left[p_{1} \theta_{1} F_{1}-\omega\right]= & \omega \frac{\dot{F}_{1}+h_{1}(t)}{F_{1}}+\left[p_{1} \theta_{1} F_{1}-\omega\right]\left[\alpha_{10}+2 \alpha_{11} F_{1}+\alpha_{12} F_{2}+\alpha_{13} F_{3}\right] \\
& +\alpha_{21} \frac{\theta_{1}}{\theta_{2}}\left[p_{2} \theta_{2} F_{2}-\omega\right] F_{1}+\alpha_{31} \frac{\theta_{1}}{\theta_{3}}\left[p_{3} \theta_{3} F_{3}-\omega\right] F_{1}
\end{aligned}
$$


Using the definition of $\dot{F}_{1}$

$$
\begin{aligned}
\delta\left[p_{1} \theta_{1} F_{1}-\omega\right]= & \omega \frac{\left[\alpha_{10}+\alpha_{11} F_{1}+\alpha_{12} F_{2}+\alpha_{13} F_{3}\right] F_{1}}{F_{1}} \\
& +\left[p_{1} \theta_{1} F_{1}-\omega\right]\left[\alpha_{10}+2 \alpha_{11} F_{1}+\alpha_{12} F_{2}+\alpha_{13} F_{3}\right] \\
& +\alpha_{21} \frac{\theta_{1}}{\theta_{2}}\left[p_{2} \theta_{2} F_{2}-\omega\right] F_{1}+\alpha_{31} \frac{\theta_{1}}{\theta_{3}}\left[p_{3} \theta_{3} F_{3}-\omega\right] F_{1} \\
= & p_{1} \theta_{1} F_{1}\left[\alpha_{10}+2 \alpha_{11} F_{1}+\alpha_{12} F_{2}+\alpha_{13} F_{3}\right]-\omega \alpha_{11} F_{1} \\
& +\alpha_{21} \frac{\theta_{1}}{\theta_{2}}\left[p_{2} \theta_{2} F_{2}-\omega\right] F_{1}+\alpha_{31} \frac{\theta_{1}}{\theta_{3}}\left[p_{3} \theta_{3} F_{3}-\omega\right] F_{1}
\end{aligned}
$$

Which implies that

$$
\begin{aligned}
0= & -2 \alpha_{11} p_{1} \theta_{1} F_{1}^{2}+\left[\theta_{1} p_{1}\left[\delta-\alpha_{10}\right]+\omega\left[\alpha_{11}+\alpha_{21} \frac{\theta_{1}}{\theta_{2}}+\alpha_{31} \frac{\theta_{1}}{\theta_{3}}\right]\right] F_{1}-\delta \omega \\
& -\theta_{1}\left[\alpha_{12} p_{1}+\alpha_{21} p_{2}\right] F_{1} F_{2}-\theta_{1}\left[\alpha_{13} p_{1}+\alpha_{31} p_{3}\right] F_{1} F_{3}
\end{aligned}
$$

Similar transformations for $\mathrm{H}_{2}$ and $\mathrm{H}_{3}$ give

$$
\begin{aligned}
0= & -2 \alpha_{22} p_{2} \theta_{2} F_{2}^{2}+\left[\theta_{2} p_{2}\left[\delta-\alpha_{20}\right]+\omega\left[\alpha_{22}+\alpha_{12} \frac{\theta_{2}}{\theta_{1}}+\alpha_{32} \frac{\theta_{2}}{\theta_{3}}\right]\right] F_{2}-\delta \omega \\
& -\theta_{2}\left[\alpha_{21} p_{2}+\alpha_{12} p_{1}\right] F_{1} F_{2}-\theta_{2}\left[\alpha_{23} p_{2}+\alpha_{32} p_{3}\right] F_{2} F_{3} \\
0= & -2 \alpha_{33} p_{3} \theta_{3} F_{3}^{2}+\left[\theta_{3} p_{3}\left[\delta-\alpha_{30}\right]+\omega\left[\alpha_{33}+\alpha_{23} \frac{\theta_{3}}{\theta_{2}}+\alpha_{13} \frac{\theta_{3}}{\theta_{1}}\right]\right] F_{3}-\delta \omega \\
& -\theta_{3}\left[\alpha_{32} p_{3}+\alpha_{23} p_{2}\right] F_{2} F_{3}-\theta_{3}\left[\alpha_{31} p_{3}+\alpha_{13} p_{1}\right] F_{1} F_{3}
\end{aligned}
$$

The last three equations specify the three unknown $F_{1}, F_{2}$, and $F_{3}$.

\section{References}

Arnason R (2006) Global warming, small pelagic fish stocks and risk. In: Hannesson R, Barange M, Herrick SF Jr (eds) Climate change and the economics of the World's fisheries: examples of small pelagic stocks. Edward Elgar, Northhampton pp 1-32

Baxter M, King RG (1999) Measuring business cycles: approximate band-pass filters for economic time series. Rev Econ Stat 81(4):575-593

Berck P, Perloff JM (1984) An open-access fishery with rational expectations. Econometrica 52(2):489-506 Bjorndal T, Munro GR (1998) The economics of fisheries management: a survey. In: Tietenberg T, Folmer H (eds) The international yearbook of environmental and resource economics 1998/1999. E. Elgar, pp 153-188

Bjornstad ON, Fromentin J-M, Stenseth NC, Gjster J (1999) Cycles and trends in cod populations. Proc Natl Acad Sci USA 96(9):5066-5071

Castilho C, Srinivasu PDN (2005) Bio-economics of a renewable resource in a seasonally varying environment. EEE Working Paper Series N. 16

Chavez FP, Ryan J, Lluch-Cota SE, Niquen CM (2003) From anchovies to sardines and back: multidecadal change in the Pacific Ocean. Science 299:217-221

Clark C, Kirkwood G (1986) On uncertain renewable resource stocks: optimal harvest policies and the value of stock surveys. J Environ Econ Manage 13(3):235-244

Costello C (2000) Renewable resource management with information on a random environment. Ph.D. Dissertation University of California at Berkeley

Costello CJ, Adams RM, Polasky S (1998) The value of El Nino forecast in the management of Salmon: a stochastic dynamic assessment. Am J Agric Econ 80(4):765-777

Costello C, Polasky S, Solow A (2001) Renewable resource management with environmental prediction. Can J Econ 34(1):196-211 
Costello C, Gaines SD, Lynham J (2008) Can catch shares prevent fisheries collapse? Science 321(5896):1678-1681

Hamilton JD (1989) A new approach to the economic analysis of nonstationary time series and the business cycle. Econometrica 57(2):357-384

Hannesson R (1983) Optimal harvesting of ecologically interdependent fish species. J Environ Econ Manage 10(4):329-345

Hannesson R, Steinshamn SI (1991) How to set catch quotas: constant effort or constant catch?J Environ Econ Manage 20(1):71-91

Hannesson R, Salvanes KG, Squires D (2008) Technological change and the tragedy of the commons: the Lofoten fishery over hundred and thirty years. Norwegian School of Economics and Business Administration. Department of Economics Discussion Paper 2008-5

Hilborn R, Walters CJ (1992) Quantitative fisheries stock assessment: choice, dynamics and uncertainty. Chapman and Hall, New York

Hollowed AB, Bax N, Beamish R, Collie J, Fogarty M, Livingston P, Pope J, Rice JC (2000) Are multispecies models an improvement on single-species models for measuring fishing impacts on marine ecosystems? ICES J Mar Sci 57(3):707-719

Jackson JBC, Kirby MX, Berger WH, Bjorndal KA, Botsford LW, Bourque BJ, Bradbury RH, Cooke R, Erlandson J, Estes JA, Hughes TP, Kidwell S, Lange CB, Lenihan HS, Pandolfi JM, Peterson CH, Steneck RS, Tegner MJ, Warner RR (2001) Historical overfishing and the recent collapse of coastal ecosystems. Science 293:629-637

Kirkley J, Paul CJM, Squires D (2002) Capacity and capacity utilization in common-pool resource industries. Environ Resour Econ 22(1-2):71-97

Lotka AJ (1925) Elements of physical biology. Williams \& Wilkins Company, Baltimore

Ludwig D, Hilborn R, Walters C (1993) Uncertainity, resource exploitation, and conservation: lessons from history. Ecol Appl 3(4):547-549

Mallet JP, Charles S, Persat H, Auger P (1999) Growth modelling in accordance with daily water temperature in European grayling (Thymallus Thymallus L.). Can J Fish Aquat Sci 56(6):994-1000

Mangel M, Marinovic B, Pomeroy C, Croll D (2002) Requiem for Ricker: unpacking MSY. Bull Mar Sci 70(2):763-781

Newell RG, Sanchirico JN, Kerr S (2005) Fishing quota markets. J Environ Econ Manage 49(3):437-462

Parma AM (1990) Optimal harvesting of fish populations with non-stationary stock-recruitment relationships. Nat Resour Model 4(1):39-76

Perman R, Ma Y, McGilvray J, Common M (2003) Natural resource and environmental economics, 3rd edn. Addison Wesley, USA

Pew Oceans Commission (2003) State of America's Oceans: charting a course for sea change. Pew Oceans Commission, Arlington

Pindyck RS (1984) Uncertainty in the theory of renewable resource markets. Rev Econ Stud 51(2):289-303

Pyper BJ, Peterman RM (1998) Comparison of methods to account for autocorrelation in correlation analyses of fish data. Can J Fish Aquat Sci 55(9):2127-2140

Reed W (1979) Optimal escapement levels in stochastic and deterministic harvesting models. J Environ Econ Manage 6(4):350-363

Ricker WE (1975) Computation and interpretation of biological statistics of fish populations. Department of the Environment, Fisheries and Marine Service, Ottawa

Roughgarden J, Smith F (1996) Why fisheries collapse and what to do about it. Proc Natl Acad Sci USA 93(10):5078-5083

Rykaczewski RR, Checkley DM (2008) Influence of ocean winds on the pelagic ecosystem in upwelling regions. Proc Natl Acad Sci U S A 105(6):1965-1970

Scott A (1955) The fishery: the objectives of sole ownership. J Polit Econ 63(2):116-124

Sethi G, Costello C, Fisher A, Hanemann M, Karp L (2005) Fishery management under multiple uncertainty. J Environ Econ Manage 50(2):300-318

Singh R, Weninger Q, Doyle M (2006) Optimal fisheries management with stock uncertainty and costly capital adjustment. J Environ Econ Manage 52(2):582-599

Smith MD, Zhang J, Coleman FC (2007) Structural modeling of marine reserves with Bayesian estimation. Mar Resour Econ 22(2):121-136

Stefanou SE, Wilen JE (1992) License values in restricted access fisheries. Bull Math Biol 54(2-3):209-218

Taylor GH (2004) Long-term climate and ocean trends and Salmon populations in the Pacific Northwest, 16 November 2004. http://www.nwcouncil.org/library/ocean/05_trends.htm

U.S. Oceans Commission (2004) A blueprint for the 21st century. U.S. Government Printing Office, Washington 
Volterra V (1931) In: Chapman RN (ed) Animal ecology, with special reference to insects. McGraw-Hill, New York, pp 409-448

Walker JM, Gardner R, Ostrom E (1990) Rent dissipation in a limited-access common-pool resource: experimental evidence. J Environ Econ Manage 19(3):203-211

Walters C, Parma AM (1996) Fixed exploitation rate strategies for coping with effects of climate change. Can J Fish Aquat Sci 53(1):148-158

Walters C, Christensen V, Pauly D (1997) Structuring dynamic models of exploited ecosystems from trophic mass-balance assessments. Rev Fish Biol Fish 7(2):139-172

Weitzman ML (2002) Landing fees vs harvest quotas with uncertain fish stocks. J Environ Econ Manage 43(2):325-338 